\title{
Probability theory and causation A branching space-times analysis
}

\author{
Thomas Müller \\ Philosophisches Seminar, LFB III, Universität Bonn \\ Lennéstr. 39, 53113 Bonn, Germany \\ Phone: +49 228 73-3786, Fax: -5066 \\ E-mail: Thomas.Mueller@uni-bonn.de
}

\begin{abstract}
We provide a formally rigorous framework for integrating singular causation, as understood by Nuel Belnap's theory of causae causantes, and objective single case probabilities. The central notion is that of a causal probability space whose sample space consists of causal alternatives. Such a probability space is generally not isomorphic to a product space. We give a causally motivated statement of the Markov condition and an analysis of the concept of screening-off.
\end{abstract}

\section{Causal dependencies and probabilities}

Probability theory describes the likelihood of outcomes of chance set-ups. In this paper, we will assume that the framework of probability theory applies to single, concrete chance set-ups such as a single coin toss at a specific place and time. This assumption appears to be uncontroversial in view of the fact that probability theory is actually successfully applied. Since we are after a metaphysical picture combining causation and probabilities, we will assume that both causal relations and single case probabilities are objective. The aim of this paper may be described as trying to make sense of that assumption by showing how it might be true.

Probability theory by itself has nothing to say about causation. However, questions of probabilities and questions of causation are obviously related. E.g., one may ask whether probabilities can be a guide to discovering causal relations. The literature on this question is large. When moved from an epistemic to a metaphysical level, the question gets a different reading: What are the objective influences of objectively existing causal relations on objectively existing probabilities? If a concrete situation involves more than one chance set-up, the causal interrelation of the set-ups may impose constraints on the probabilistic structure of the situation. E.g., if depending on the outcome of one chance set-up, another one is enabled or not, that causal structure should be mirrored in the probabilistic structure. 
In this paper, we will provide a rigorous framework for bringing together causation and probabilities. The paper is based on an existing theory of causation Nuel Belnap's theory of causae causantes (Belnap, 2003b), the background for which is branching space-times (Belnap, 1992). In this paper, we presuppose the notation of Belnap (2003b), which should be consulted. A very readable introduction to the problem of bringing together causation and probabilities is given in Weiner and Belnap (2004), which should also be consulted. The present paper may be read as a sequel to that paper, developing its ideas in more formal detail. ${ }^{1}$

\subsection{Background: Causation in branching space-times}

Branching space-times is a rigorous framework for combining relativistic spacetimes with indeterminism. The basic building blocks of branching space-times are a set $W$ of concrete point events and a partial order $\leq$ defined on $W$; the companion strict order is written " $<$ ". Histories $h$ are maximal directed sets, i.e., maximal such that for any $e_{1}, e_{2} \in h$ there is $e_{3} \in h$ with $e_{1}<e_{3}$ and $e_{2}<e_{3}$. Points $e_{1}, e_{2}$ that are incomparable (neither $e_{1} \leq e_{2}$ nor $e_{2}<e_{1}$ ) can still be elements of the same history, viz., if they have a common upper bound. In that case, we call $e_{1}$ and $e_{2}$ space-like related, and we write $e_{1} \operatorname{SLR} e_{2}$. - Branching occurs at single indeterministic points. Thus, any two histories $h_{1}, h_{2}$ to which an indeterministic point $e$ belongs are either divided at $e\left(h_{1} \perp_{e} h_{2}\right)$ or undivided at $e\left(h_{1} \equiv_{e} h_{2}\right)$, the latter being an equivalence relation. The set of histories containing $e$ is written $H_{(e)} . H_{(e)}$ is partitioned by $\equiv_{e}$; we write $\Pi_{e}$ for the set of partitions and $\Pi_{e}\langle h\rangle$ for the unique element of $\Pi_{e}$ containing $h$ (presupposing $e \in h$ ). Thus, $\Pi_{e}\langle h\rangle$ is the set of histories containing $e$ that are undivided from $h$ at $e$. An initial $I$ is a set of point events that are part of one history. We write $H_{[I]}$ for the set of histories of which $I$ is a part; $H_{[I]} \neq \emptyset$. It is often adequate to think of $I$ as an upper bounded initial chain, and $I=\{e\}$ is an important special case. (A chain is a set of pairwise comparable point events.) The primary propositional outcomes of an initial $I$ are the elements of the partition $\Pi_{I}$ induced by undividedness at $I$, where $h \equiv_{I} h^{\prime}$ iff $h \equiv \equiv_{e} h^{\prime}$ for all $e \in I$. An outcome event $O$ is a set of (lower bounded) outcome chains, all of which begin in one history. We write $H_{\langle O\rangle}$ for the set of histories in which $O$ begins to occur (even though it does not have to occur as a whole); by definition, $H_{\langle O\rangle} \neq \emptyset$.

\footnotetext{
${ }^{1}$ Most of this paper was written while I was a guest of Nuel Belnap's at the University of Pittsburgh. I thank him both for his hospitality and for many helpful discussions. Furthermore, I have greatly benefitted from his numerous comments on previous versions of the paper, including the suggestion to "try the contrapositive" on a previous proof of Lemma 2. I also thank Tomasz Placek for many stimulating discussions and for specific comments. Nuel Belnap, Tomasz Placek and Matthew Weiner have kindly shared their results prior to publication. Support by the Deutsche Forschungsgemeinschaft is also gratefully acknowledged.
} 
Subscribing to the rather uncontroversial assumption that causation is a twoplace relation between cause and effect, the following two questions are crucial for any theory of causation:

1. What is caused? Which entities are effects?

\section{What causes? Which entities are causes?}

As is well known, there are numerous different answers to these questions. One main divide is between singular and generic causes and effects. Obviously, both notions are important - consider "he broke the vase" vs. "smoking causes cancer". It seems both ontologically most promising and most in line with our framework to address questions of singular causation first. Thus we look for singular, concrete causes and effects. What sorts of entities are these? A good intuition seems to be that causation involves change, but it is notoriously difficult to say what a change is. The notion of a transition, introduced by von Wright (1963), is a liberalized notion of change: A transition is "something and then something"; not necessarily "... something else". Following von Wright, in causation in branching space-times the crucial causal notion is therefore that of a transition. Technically, a transition, written $I \longmapsto O$, is just an ordered pair of sets $I$ and $O$ of point events, where $I$ is an initial event and $O$ is an outcome event located after $I$ (i.e., for all $e_{I} \in I$ and for all $e_{O} \in O$ we have $e_{I}<e_{O}$ ).

The answer to question (1) above, from the point of view of causation in branching space-times, is accordingly:

\section{Transitions are caused.}

In sharp contrast to other theories of causation, causation in branching space-times assumes that non-trivial causation depends on indeterminism. A transition $I \longmapsto O$ whose outcome $O$ is bound to occur, given that $I$ occurs, has no causes. It does not need any, since it happens anyway. Causes are thus understood as originating causes (causae causantes), which seems to capture our actual usage of the category of causation much more nicely than other accounts.

What are the causes? Causation in branching space-times gives the following answer to question (2) above:

2. Causes are sets of (especially simple) transitions.

Thus, a transition is caused by (possibly a huge number of) transitions. The transitions that figure as causes are of an especially simple sort: they are from a single point $e$ as initial (a past cause-like locus of the caused transition) to one of $e$ 's primary outcomes $\Pi_{e}\langle h\rangle$. Given an outcome event $O$ of $e, e<O$, the set of histories 
$\Pi_{e}\langle O\rangle$ is the uniquely determined member of the partition $\Pi_{e}$ of $H_{(e)}$ in which $O$ is kept possible, i.e., for which $\Pi_{e}\langle O\rangle \cap H_{\langle O\rangle} \neq \emptyset$. In fact, we have $H_{\langle O\rangle} \subseteq \Pi_{e}\langle O\rangle$. The corresponding transitions, written $t_{i}=e_{i} \longmapsto H_{i}, H_{i} \in \Pi_{e_{i}}$, are called basic transitions. It turns out, as it should, that basic transitions are their own causes. Thus, they may figure as irreducibly indeterministic building blocks of our indeterministic world. In the theory that will be developed in this paper, they will also form the building blocks for the construction of probability spaces, thus providing a close link between causation and probabilities.

Having mentioned the concepts, we now give the formal definitions of "past cause-like locus" and "causa causans" (cf. Belnap 2003b) for later reference.

Definition 1 (Past cause-like loci) Let $I \longmapsto O$ be a transition. Then the set of past cause-like loci for that transition, $\operatorname{pcl}(I \longmapsto O)$, is defined to be

$$
\operatorname{pcl}(I \longmapsto O):=\left\{e \in W \mid e<O \wedge \exists h \in H_{[I]} h \perp_{e} H_{\langle O\rangle}\right\} .
$$

Thus, a point event $e$ is a past cause-like locus for $I \longmapsto O$ if (i) $e<O$, i.e., $e$ is a lower bound for the outcome $O$, and (ii) there is a history $h$ in which the initial $I$ finishes that is separated at $e$ from all histories in which $O$ begins to occur. Past cause-like loci make a difference as to the occurrence of $O$. They are therefore necessarily indeterministic points, i.e., points with more than one immediate outcome.

Definition 2 (Causae causantes) Let $I \longmapsto O$ be a transition. Then the set of causae causantes for that transition, $c c(I \longmapsto O)$, is defined to be

$$
c c(I \longmapsto O):=\left\{e \longmapsto \Pi_{e}\langle O\rangle \mid e \in \operatorname{pcl}(I \longmapsto O)\right\} .
$$

A causa causans for a transition $I \longmapsto O$ is thus a transition from a past cause-like locus $e$ to that unique immediate outcome $\Pi_{e}\langle O\rangle$ of $e$ that keeps $O$ possible right after $e$.

\subsection{What are probabilities defined for?}

Intuitively, probabilities are probabilities of outcomes of chance set-ups. Causation in branching space-times offers the notion of an indeterministic transition to stand for "chance set-up". If $I \longmapsto O$ can occur, but $O$ does not have to occur given $I$, then it will in many cases be sensible to ask how probable the transition $I \longmapsto O$ was compared to the alternatives. Thus, probabilities are probabilities of transitions. (In the causally trivial case of a deterministic transition, the probability will be trivially equal to one.) We will bind the notion of probabilities fully to the notion of causae 
causantes: Since the framework has room for indeterminism, it should be possible to grade that indeterminism numerically at least in some cases. ${ }^{2}$

We will reduce all considerations of probabilities of transitions to considerations of probabilities of combinations of basic transitions. This will be done by in some way identifying a transition with its set of causae causantes. We call two transitions causally equivalent $\left(I \longmapsto O \stackrel{c}{\equiv} I^{\prime} \longmapsto O^{\prime}\right.$ ) iff they have the same causae causantes:

\section{Definition 3 (Causal equivalence of transitions)}

$$
I \longmapsto O \stackrel{c}{=} I^{\prime} \longmapsto O^{\prime} \quad \text { iff } \quad c c(I \longmapsto O)=c c\left(I^{\prime} \longmapsto O^{\prime}\right) .
$$

The relation $\stackrel{c}{\equiv}$ is an equivalence relation, and an equivalence class may be specified via the set of causae causantes of one of its members. In what follows, we will therefore not talk about transitions in general, but about sets of basic transitions. ${ }^{3}$ The main work that is to be done is to find out how basic transitions combine and which probabilities should be assigned to such combinations.

Thus, the next section introduces some facts about basic transitions, and the section after that is concerned with probabilities.

\section{Basic transitions}

\subsection{Basics of basic transitions}

Basic transitions are transitions from a point event (initial) $e_{i}$ to one of $e_{i}$ 's immediate outcomes $H_{i} \in \Pi_{e_{i}}$; we write

$$
t_{i}=e_{i} \longmapsto H_{i}
$$

The different $H_{i} \in \Pi_{\left(e_{i}\right)}$ partition $H_{\left(e_{i}\right)}$, the set of all histories containing $e_{i}$. Points $e_{i}$ for which $\Pi_{\left(e_{i}\right)}$ has only one member are called trivial; at such points, no splitting occurs. A transition $e_{i} \longmapsto H_{\left(e_{i}\right)}$ with a trivial initial is also called trivial. We will mostly be interested in non-trivial, or indeterministic, points and

\footnotetext{
${ }^{2}$ It seems to be rather uncontroversial that probabilities are numerically graded modalities; cf. e.g., Popper $(1982,70)$ and van Fraassen $(1980,198)$. Once possibilities are there, probabilities are (well, almost) for free.

${ }^{3}$ This does not mean that all that is important about a transition is its set of causae causantes. E.g., epistemically the spatiotemporal location of $I$ and $O$ may play a huge role, and there are also important differences between a basic transition and a non-basic transition that is causally equivalent to it. All we claim in this paper is that on the ontological level, all that is probabilistically important about a transition is in its causae causantes, taken together.
} 
transitions. A cause-like locus is always indeterministic by definition, so sets of causae causantes consist of non-trivial basic transitions.

We call two basic transitions $t_{1}=e_{1} \longmapsto H_{1}$ and $t_{2}=e_{2} \longmapsto H_{2}$ initialequivalent if their initials are the same $\left(e_{1}=e_{2}\right)$; in this case, we write $t_{1} \stackrel{i}{\equiv} t_{2}$. This is an equivalence relation, and the equivalence class of $t_{i},\left[t_{i}\right]$, is the set of all basic transitions with initial $e_{i}$. Since $\left[t_{i}\right]$ is completely specified by the initial $e_{i}$, we also write the equivalence classes as $\left[e_{i}\right]:^{4}$

$$
\left[t_{i}\right]=\left[e_{i}\right]=\left\{e_{i} \longmapsto H \mid H \in \Pi_{e_{i}}\right\} .
$$

We call an initial $e_{i}$ finitely splitting iff $\left[e_{i}\right]$ is finite. - On the set of basic transitions, we can define a partial ordering relation as follows:

$$
t_{i}<t_{j} \quad \text { iff } \quad e_{i}<e_{j} \text { and } H_{\left(e_{j}\right)} \subseteq H_{i} .^{5}
$$

Thus, the transition $t_{j}$ is above $t_{i}\left(t_{i}<t_{j}\right)$ if $t_{j}$ can occur in the outcome $H_{i}$ of $t_{i}$. For this it is not enough that $e_{i}<e_{j}$ : Since at $e_{i}$, histories split, $e_{j}$ must also occur in the outcome $H_{i}$ of $e_{i}$.

The ordering of transitions sheds some light on when two transitions are compatible. This will now be spelled out in terms of consistent sets of transitions.

\subsection{Set of basic transitions}

Sets of basic transitions will be the basic building blocks for the probability spaces to be defined later on: they will constitute the elements of the sample space. Not all sets of basic transitions will do, however. Some sets are such that they cannot occur in one history - they are causally impossible. The most useful sets of basic transitions are the consistent ones, where consistency is something like causal possibility.

\subsubsection{Consistency of sets of basic transitions}

The notion of consistency is defined as follows:

Definition 4 (Consistent set of basic transitions) A set $T=\left\{t_{1}, t_{2}, \ldots\right\}$ of basic transitions ( $t_{i}=e_{i} \longmapsto H_{i}$ ) is consistent iff all the outcomes have a history in

\footnotetext{
${ }^{4}$ This notion of initial-equivalence of basic transitions is to be distinguished from the notion of causal equivalence of transitions employed above. Note also that on $\left[t_{i}\right]$, probabilities can be easily defined, since we can introduce a natural set-theoretic Boolean algebra structure on $\Pi_{e_{i}}$. This will be our starting point later on.

${ }^{5}$ Note that given $e_{i}<e_{j}$, if $H_{\left(e_{j}\right)} \cap H_{i} \neq \emptyset$, then $H_{\left(e_{j}\right)} \subseteq H_{i}$ by transitivity of $\equiv_{e_{i}}$.
} 
common, i.e., iff $\cap_{t_{i} \in T} H_{i} \neq \emptyset$. (The empty set $\emptyset$ is considered consistent, since the intersection of an empty set of histories, $\cap_{t_{i} \in \emptyset} H_{i}$, is by definition taken to be the set $\mathfrak{H}$ of all histories.)

By this definition, any subset of a consistent set of basic transitions is consistent. A good thing to note is that the set of basic transitions that we identified with a general transition $I \longmapsto O$ is consistent in the sense just defined.

Lemma 1 For any transition $I \longmapsto O$, the set of its causae causantes is consistent.

Proof: Let $I \longmapsto O$ be given, and let $T=c c(I \longmapsto O)$. By definition, any $e_{i} \longmapsto$ $H_{i}=t_{i} \in T$ satisfies $e_{i}\left\langle O\right.$ and $H_{i}=\Pi_{\left(e_{i}\right)}\langle O\rangle$, so that $H_{\langle O\rangle} \subseteq H_{i}$. Thus, $H_{\langle O\rangle} \subseteq \cap_{t_{i} \in T} H_{i}$. As $H_{\langle O\rangle} \neq \emptyset, T$ is consistent.

Before we can move on, we need to state, and assume, an important postulate and a convenient limitation.

\subsubsection{Limitations: Finiteness and exclusion of modal funny business}

The theory to be developed here will be limited in two ways. The first limitation is for convenience's sake and will have to be removed in a further development of the theory: We will assume that all sets of causae causantes and all probability structures that we are going to deal with are finite. This is by no means a "natural" limitation, i.e., we do not assume that there are any deep ontological reasons for this limitation. It is just that sticking to the finite case will allow us to focus on the problem of combining causality and probability without having to cope with problems of infinite probability structures all along. ${ }^{6}$

The second limitation is fundamental. In the general theory of branching spacetimes, there may occur a phenomenon that Nuel Belnap has aptly called "modal funny business": roughly, some combinatorially possible histories may be absent. Since we rely on smooth combinatorics, we will have to exclude models involving modal funny business from our considerations. This seems not to be much of a constraint, however, since a modally "funny" model may be mimicked probabilistically by a model in which the combinatorics are smooth, and formerly missing histories are excluded via probability zero.

Belnap (2003a) proves the equivalence of four different notions of modal funny business. For our purposes, it is best to use the following notion:

Definition 5 (Modal funny business) In a model of branching space-times, there is primary space-like related modal funny business iff there are two initial events

\footnotetext{
${ }^{6} \mathrm{Cf}$. note 8 below for some comments on infinite structures.
} 


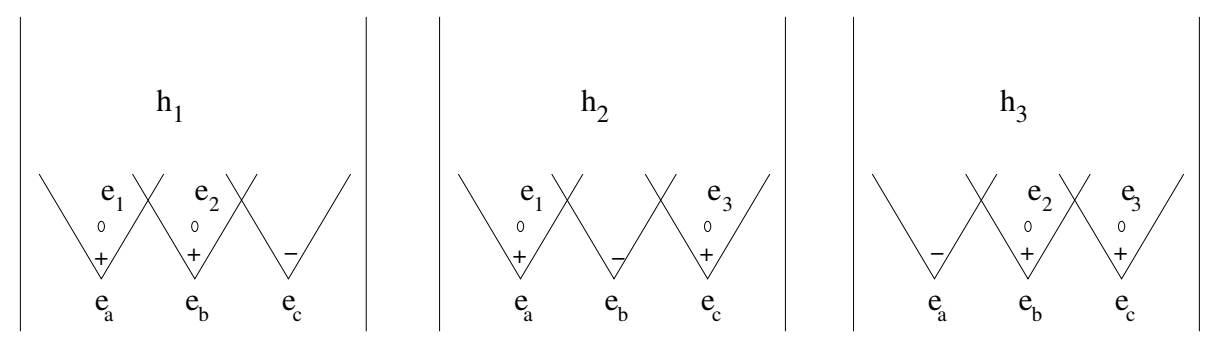

Figure 1: Effects of modal funny business.

(nonempty sets of point events that are part of one history) $\mathbf{I}_{1}, \mathbf{I}_{2}$ such that $\mathbf{I}_{1}$ SLR $\mathbf{I}_{2}$ $\left(\forall e_{1} \in \mathbf{I}_{1} \forall e_{2} \in \mathbf{I}_{2} e_{1}\right.$ SLR $\left.e_{2}\right)$, but one primary outcome $\Pi_{I_{1}}\left\langle h_{1}\right\rangle$ of $\mathbf{I}_{1}$ has an empty intersection with one primary outcome $\Pi_{I_{2}}\left\langle h_{2}\right\rangle$ of $\mathbf{I}_{2} \cdot{ }^{7}$

Figure 1 illustrates what may happen if there is modal funny business. The three depicted histories are all the histories there are in the model. Consider first the events $e_{a}, e_{b}$, and $e_{c}$ that occur in all three histories. Each of these is an indeterministic point event with two outcomes, which are labeled ' + ' and '-'. There is modal funny business according to Definition $5-$ e.g., for $\mathbf{I}_{1}=\left\{e_{a}\right\}, \mathbf{I}_{2}=\left\{e_{b}\right\}$, the intersection of the '-' outcomes is empty; among the three histories, none contains both $e_{a} \longmapsto-$ and $e_{b} \longmapsto-$. In the depicted case, there are also three pairwise space-like related points $e_{1}, e_{2}$, and $e_{3}$ which are nonetheless not all contained in one single history: Each pair, e.g., $e_{1}, e_{2}$, is incomparable, and there is a history, e.g., $h_{1}$, containing both points, witnessing the space-like relatedness. But contrary to what one might assume, none of the three histories of the model contains all three points $e_{1}, e_{2}$, and $e_{3}$. In the absence of modal funny business, such a situation is ruled out: Given no modal funny business according to Definition 5, for any finite set of pairwise SLR points there is a history containing the whole set, and any set of transitions with pairwise SLR initials has a nonempty common outcome.

Lemma 2 If there is no modal funny business according to Definition 5, the following holds: (a) Let $E=\left\{e_{1}, \ldots, e_{n}\right\}$ be such that for $e_{i}, e_{j} \in E$, if $e_{i} \neq e_{j}$, then $e_{i} \operatorname{SLR} e_{j}$. Then $H_{[E]} \neq \emptyset$, i.e., there is a history containing all the points in E. (b) Furthermore, let $T=\left\{t_{1}, \ldots, t_{n}\right\}$ be a set of transitions $\left(t_{i}=e_{i} \longmapsto H_{i}\right)$ such that the set of initials $E=\left\{e_{i} \mid t_{i} \in T\right\}$ fulfills the conditions of (a) and such that no two transitions from $T$ have the same initial. Then $T$ is consistent, i.e., the transitions in $T$ have a common outcome: $\cap_{t_{i} \in T} H_{i} \neq \emptyset$.

\footnotetext{
${ }^{7}$ In the infinite case, this definition will have to be altered; cf. note 8 below for some indications of what needs to be changed.
} 
Proof: We prove only (b), from which one may obtain (a) as follows: if only $E$ is given, select arbitrary outcomes $H_{i} \in \Pi_{e_{i}}$ for each $e_{i} \in E$. If then $\cap_{t_{i} \in T} H_{i} \neq \emptyset$, we have $H_{[E]} \neq \emptyset$ since $H_{i} \subseteq H_{\left(e_{i}\right)}$. - So assume that the premises of (b) hold, but that $\cap_{t_{i} \in T} H_{i}=\emptyset$. Let $T^{\prime} \subset T$ be a subset of $T$ that is maximal with respect to $\cap_{t_{i} \in T^{\prime}} H_{i} \neq \emptyset$. Now let $t_{j} \in T-T^{\prime}$. Set $E^{\prime}=\left\{e_{i} \mid t_{i} \in T^{\prime}\right\}$. By assumption, the elements of $E$ are pairwise SLR, so the sets $I_{1}=E^{\prime}, I_{2}=\left\{e_{j}\right\}$ are initials, and the sets $\cap_{t_{i} \in T^{\prime}} H_{i}$ and $H_{j}$ are primary outcomes, fulfilling the requirement of Definition 5. Now since there is no modal funny business by assumption, that Definition implies $\cap_{t_{i} \in T^{\prime}} H_{i} \cap H_{j} \neq \emptyset$, contradicting the maximality of $T^{\prime}$.

In what follows, we will assume that there is no modal funny business:

Postulate 1 (No modal funny business) There is no modal funny business according to Definition 5.

\subsubsection{Characterization of consistency in the absence of modal funny business}

Given finite structures and no modal funny business, the notion of consistency of a set of basic transitions can be expressed in different ways:

Lemma 3 Let $T=\left\{t_{1}, \ldots, t_{n}\right\}, t_{i}=e_{i} \longmapsto H_{i}$. The following conditions are equivalent:

1. $T$ is consistent.

2. There is a history to which all the $e_{i}$ belong $\left(\cap_{t_{i} \in T} H_{\left(e_{i}\right)} \neq \emptyset\right)$. Furthermore, (a) if $e_{i}=e_{j}$, then $H_{i}=H_{j}$, and (b) if $e_{i}<e_{j}$, then $H_{\left(e_{j}\right)} \subseteq H_{i}$.

3. For any $t_{i}, t_{j} \in T$, exactly one of the following holds:

(a) $e_{i}=e_{j}$ and $H_{i}=H_{j}\left(\right.$ i.e., $\left.t_{i}=t_{j}\right)$ or

(b) $t_{i}<t_{j}$ or

(c) $t_{j}<t_{i}$ or

(d) $e_{i} \operatorname{SLR} e_{j}$.

Proof: $1 \Rightarrow 2$ : For all $t_{i}, H_{i} \subset H_{\left(e_{i}\right)}$, and $\cap_{t_{i} \in T} H_{i} \neq \emptyset$ by (1). So there is a history to which all the $e_{i}$ belong. If $e_{i}=e_{j}$, then $H_{i} \cap H_{j} \neq \emptyset$ iff $H_{i}=H_{j}$ (the family of outcomes of $e_{i}$ is a partition of $\left.H_{\left(e_{i}\right)}\right)$. If $e_{i}<e_{j}$, then $H_{\left(e_{j}\right)} \cap H_{i}=\emptyset$ would imply $H_{j} \cap H_{i}=\emptyset$ (since $H_{j} \subseteq H_{\left(e_{j}\right)}$ ), meaning that $T$ would be inconsistent.

$2 \Rightarrow 3$ : Assume $T$ violates (3), i.e., there are $t_{i}, t_{j} \in T$ such that none of (3a)-(3d) applies. This is possible in four ways: (a) $e_{i}=e_{j}$, but $H_{i} \neq H_{j}$ - this 
violates (2); (b) $e_{i}<e_{j}$, but $H_{\left(e_{j}\right)} \cap H_{i}=\emptyset$ - again violating (2); (c) for $e_{j}<e_{i}$ symmetrical to (b); (d) $e_{i} \nless e_{j}$ and $e_{j} \not \leq e_{i}$ and not $e_{i} \operatorname{SLR} e_{j}$ - this means that there is no history to which both $e_{i}$ and $e_{j}$ belong, again violating (2).

$3 \Rightarrow 1$ : Let $T^{\prime}:=\left\{t_{i} \in T \mid \neg \exists t_{j} \in T t_{i}<t_{j}\right\}$ be the set of maximal elements of $T$. It will be sufficient to establish that $\cap_{t_{i} \in T^{\prime}} H_{i} \neq \emptyset$, since if $t_{i}<t_{j}$, $H_{j} \subseteq H_{i}$. Now for $t_{i}, t_{j} \in T^{\prime}$, if $t_{i} \neq t_{j}$, then we have $e_{i} \operatorname{SLR} e_{j}: e_{i}=e_{j}$ is ruled out by (3a), and $t_{i}<t_{j}$ or $t_{j}<t_{i}$ is impossible since $T^{\prime}$ is the set of the maximal elements. Thus we have a set of pairwise space-like related points with outcomes $H_{i}$ specified, and by Lemma 2 (b), there is a history in the intersection of all outcomes.

The following Lemma characterizes the special status of the maximal elements: their outcomes may be changed at will without violating consistency.

Lemma 4 If $T=\left\{t_{1}, \ldots, t_{n}\right\}$ is consistent and $t_{0} \in\left[t_{i}\right], t_{0} \neq t_{i}$, then $T^{\prime}=$ $\left(T-\left\{t_{i}\right\}\right) \cup\left\{t_{0}\right\}$ is consistent iff $t_{i}$ is maximal in $T$.

Proof: " $\Leftarrow$ ": If $t_{i}$ is maximal, then the set with $t_{0}$ substituted is also consistent by Lemma 3 (2).

" $\Rightarrow$ ": Let $t_{i}$ not be maximal in $T$, i.e., there is $t_{j} \in T$ s.t. $t_{i}<t_{j}$, i.e., $e_{i}<e_{j}$ and $H_{\left(e_{j}\right)} \cap H_{i} \neq \emptyset$. Since $\Pi_{e_{i}}$ is a partition of $H_{\left(e_{i}\right)}, H_{\left(e_{j}\right)} \cap H_{0}=\emptyset$. By Lemma 3 (2), the set with $t_{0}$ substituted is thus inconsistent.

\section{Causal probability theory}

We have now prepared the ground for the introduction of probabilities into the framework of causation in branching space-times. By the close link between causation and probability that was assumed at the outset, the objects that figure as causes - sets of basic transitions - will be the objects on which probabilities will be defined. In order to have probabilities, we need probability spaces:

Definition 6 (Probability space) $A$ probability space is a triple $\langle A, F, \mu\rangle$, where $A$ is a nonempty set (the "sample space"), $F$ is a Boolean $\sigma$-algebra (sometimes called "event algebra") of subsets of $A$ (with zero element $\mathbf{0}=\emptyset$ and unit element $\mathbf{1}=A$ ), and $\mu$ is a normalized, countably additive measure on $F$, i.e., (i) $\mu(\mathbf{1})=1$ and (ii) if $\left(f_{i}\right)_{i \in I}$ is an at most countable family of disjoint elements of $F$, then $\mu\left(\cup_{i \in I} f_{i}\right)=\sum_{i \in I} \mu\left(f_{i}\right)$.

As stated above, we will consider finite probability structures exclusively. Thus, we will assume in the following that all probability spaces that we deal with are finite. 
In a finite probability space, $F$ is just the usual set-theoretic algebra of subsets of $A$, with the elements of $A$ playing the role of atoms, or basic alternatives.

The basic questions of this section may be characterized as follows:

Given a transition $I \longmapsto O$, represented by a consistent set $T$ of basic transitions: In which probability space can we speak of a "probability for $T$ "? That is:

1. What is the sample space $A$ that contains $T$ and its causal alternatives?

2. What is the Boolean $\sigma$-algebra $F$ ?

3. What is the measure $\mu$; which general constraints hold for $\mu$ ?

4. How do the measures in different probability spaces relate?

We will motivate our answers to these questions through a number of increasingly complex examples.

\subsection{Examples for $T$}

The simplest example is given by a trivial transition: its set of causae causantes is empty. In this case, we have $A=\{\emptyset\}, F$ consists of two elements only ( $A$ and the empty set), and $\mu(A)=1$. The first non-trivial example is given by a singleton set of transitions.

\subsection{1 $T=\left\{t_{1}\right\}$}

In this case, $t_{1}$ is a basic transition with initial $e_{1}$ and outcome $H_{1} \in \Pi_{\left(e_{1}\right)}$. The probability space that needs to be considered is obvious:. The sample space is naturally taken to be $\left[t_{1}\right]$, i.e., the set of all basic transitions with initial $e_{1}$. Our finiteness assumption requires that $\left[t_{1}\right]$ be finite. This is not guaranteed, and it is certainly not adequate in all situations, but there exist well-known measure-theoretic ways of dealing with infinities in this case (cf. note 8 below).

Given finite $A=\left[t_{1}\right], F$ is just the set of all subsets of $A$. The measure $\mu$ is uniquely characterized by its value on the singleton sets, since any member of $F$ is the disjoint union of a finite number of singletons. This is all standard probability theory, with no hint of causal constraints.

\subsection{2 $T=\left\{t_{1}, t_{2}\right\}, e_{1} \operatorname{SLR} e_{2}$}

The simplest case of a set $T$ with two elements is the case in which the initials are space-like separated. (We do not consider the case that $T$ is inconsistent at the 
moment, since that cannot happen for $T$ derived from a given transition $I \longmapsto O$, cf. Lemma 1.)

In the present case, $T$ combines two transitions. For single transitions, we already know how to construct probability spaces - simply take the set $\left[t_{i}\right]$ to be the sample space, as outlined above. From the two probability spaces $P_{1}=$ $\left\langle A_{1}, F_{1}, \mu_{1}\right\rangle$ and $P_{2}=\left\langle A_{2}, F_{2}, \mu_{2}\right\rangle$ we may then form the product space, $P=$ $\langle A, F, \mu\rangle$, where $A=A_{1} \times A_{2}$ is the Cartesian product of $A_{1}$ and $A_{2}$, i.e., the set of ordered pairs $\left\langle a_{1}, a_{2}\right\rangle$ with $a_{i} \in A_{i}, i=1,2$, and $F=F_{1} \times F_{2}$ is the respective product Boolean algebra. For the measure $\mu$, we at least require the so-called marginal property:

Definition 7 (Marginal property for pairs) A measure $\mu$ on the product Boolean algebra $F=F_{1} \times F_{2}$ has the marginal property iff (i) for all $f_{1} \in F_{1}, \mu\left(\left\langle f_{1}, \mathbf{1}_{2}\right\rangle\right)=$ $\mu_{1}\left(f_{1}\right)$ and (ii) for all $f_{2} \in F_{2}, \mu\left(\left\langle\mathbf{1}_{1}, f_{2}\right\rangle\right)=\mu_{2}\left(f_{2}\right)$.

The marginal property means that if one ignores the outcome of one initial by considering the certain outcome $\mathbf{1}_{i}=A_{i}$ (for which $\mu_{i}\left(\mathbf{1}_{i}\right)=1$ ), one gets back the single probability. Requiring that $\mu$ satisfy the marginal property does not fix $\mu-$ there are many ways open. One specific way is taking the product measure $\mu_{\times}$:

$$
\mu_{\times}\left(\left\langle f_{1}, f_{2}\right\rangle\right)=\mu_{1}\left(f_{1}\right) \cdot \mu_{2}\left(f_{2}\right) .
$$

The other way is to allow for correlations between outcomes of $e_{1}$ and $e_{2}$ by using a different measure.

Here comes a crucial question: Given that $e_{1}$ and $e_{2}$ are space-like related (and thus, causally separated), should we require that $\mu=\mu_{\times}$? We believe that no, since quantum-mechanical correlation experiments give strong evidence that there can be correlations between outcomes of space-like separated events. In order to remain flexible, our theory should allow both for correlations and for the product measure.

The product construction described above is standard in probability theory. A different perspective on that construction will prove to be illuminating when it comes to generalizations. By Lemma 2, any combination of one outcome of $e_{1}$ and one outcome of $e_{2}$ is consistent: Each $\left\{t_{1}^{\prime}, t_{2}^{\prime}\right\}$ with $t_{1}^{\prime} \in\left[t_{1}\right], t_{2}^{\prime} \in\left[t_{2}\right]$, is consistent. We may base our probability space on such sets and define:

$$
A=\left\{\left\{t_{1}^{\prime}, t_{2}^{\prime}\right\} \mid t_{1}^{\prime} \in\left[t_{1}\right], t_{2}^{\prime} \in\left[t_{2}\right]\right\} .
$$

By the assumption of finitude, $F$ may again be the usual set algebra, and it will suffice to define the measure $\mu$ on the atoms, i.e., on the elements of $A$. Since in this construction, we did not start with "single" probability spaces $P_{1}$ and $P_{2}$, 
we cannot state the marginal property as before. However, we may require some form of marginal property once two "single" probability spaces with respective measures $\mu_{1}$ and $\mu_{2}$ are given in addition:

Definition 8 (Marginal property for sets) Let $F$ be the Boolean algebra of sets of transitions as defined above. A measure $\mu$ on $F$ has the marginal property iff (i) for all $a_{1} \in A_{1}, \mu\left(\left\{\left\{a_{1}, a_{2}\right\} \mid a_{2} \in A_{2}\right\}\right)=\mu_{1}\left(\left\{a_{1}\right\}\right)$ and (ii) for all $a_{2} \in A_{2}$, $\mu\left(\left\{\left\{a_{1}, a_{2}\right\} \mid a_{1} \in A_{1}\right\}\right)=\mu_{2}\left(\left\{a_{2}\right\}\right)$.

This is quite similar to Definition 7: Since

$$
\mu\left(\left\{\left\{a_{1}, a_{2}\right\} \mid a_{2} \in A_{2}\right\}\right)=\sum_{a_{2} \in A_{2}} \mu\left(\left\{\left\{a_{1}, a_{2}\right\}\right\}\right),
$$

requiring that that sum be equal to $\mu_{1}\left(\left\{a_{1}\right\}\right)$ again means that by ignoring the outcome of $e_{2}$ (by summing over all possibilities), one gets back the "single" measure.

In the present case $\left(e_{1} \operatorname{SLR} e_{2}\right)$, the two mentioned perspectives are provably equivalent, since there is an isomorphism between the two mentioned probability spaces, via

$$
\left\langle a_{1}, a_{2}\right\rangle \leftrightarrow\left\{a_{1}, a_{2}\right\}
$$

The important question is which of the two perspectives generalizes. In classical probability theory, combinations of chance set-ups are adequately described via product constructions. It turns out, however, that once causal notions enter the picture, product constructions are no longer adequate. This is shown by the next example.

\subsection{3 $T=\left\{t_{1}, t_{2}\right\}, t_{1}<t_{2}$}

Apart from the case $e_{1}$ SLR $e_{2}$, which has already been considered, and the case $t_{2}<t_{1}$, which is symmetrical to the present one, this is the only possible case of a consistent set of two transitions (cf. Lemma 3 (3)). As in the previous case, two chance set-ups are combined. The attempt to apply a product construction will result in an instructive failure.

As in the case for $e_{1} \operatorname{SLR} e_{2}$, one may try to start from two probability spaces $\left\langle A_{i}, F_{i}, \mu_{i}\right\rangle$ with $A_{i}=\left[t_{i}\right], i=1,2$. The product space $\langle A, F, \mu\rangle$ is definable as before. However, that probability space is not adequate for the causal situation assumed. Consider a pair $\left\langle t_{1}^{\prime}, t_{2}^{\prime}\right\rangle$ with $t_{1} \in\left[t_{1}\right], t_{1}^{\prime} \neq t_{1}, t_{2}^{\prime} \in\left[t_{2}\right]$. That pair corresponds to a causally impossible "alternative" to $T$, since by Lemma 4 , there is no history in which both the outcome $H_{1}^{\prime}$ of $t_{1}^{\prime}$ and the initial $e_{2}$ of $t_{2}^{\prime}$ occur together. The only reasonable thing to do probabilistically seems to be to require 
that $\mu\left(\left\langle t_{1}^{\prime}, t_{2}^{\prime}\right\rangle\right)=0$ in that case (i.e., in case $\left.t_{1}^{\prime} \neq t_{1}\right)$. But then, given the marginal property, we get

$$
\mu_{1}\left(t_{1}^{\prime}\right)=\mu\left(\left\langle t_{1}^{\prime}, \mathbf{1}_{2}\right\rangle\right)=\sum_{t_{2}^{\prime} \in\left[t_{2}\right]} \mu\left(\left\langle t_{1}^{\prime}, t_{2}^{\prime}\right\rangle\right)=0 \quad \text { for } \quad t_{1}^{\prime} \neq t_{1},
$$

and by normalization of $\mu_{1}$, we get $\mu_{1}\left(t_{1}\right)=1$ : $e_{1}$ is bound to have outcome $H_{1}$. That is certainly not warranted.

Consider a real life example: Assume that at $e_{1}$ you decide at random whether to go to the races or not (possibly by flipping a fair coin); $\left[e_{1}\right]=\left\{e_{1} \longmapsto H_{1}^{1}, e_{1} \longmapsto\right.$ $\left.H_{1}^{2}\right\}, \mu(\mathrm{go})=\mu_{1}\left(e_{1} \longmapsto H_{1}^{1}\right)=0.5$. If you are at the races $\left(e_{2}\right.$ such that $e_{1}<e_{2}$ and $\left.H_{\left(e_{2}\right)} \subseteq H_{1}^{1}\right)$, you either win $\left(e_{2} \longmapsto H_{2}^{1}\right)$ or you lose $\left(e_{2} \longmapsto H_{2}^{2}\right)$; suppose $\mu_{2}($ win $)=\mu_{2}\left(e_{2} \longmapsto H_{2}^{1}\right)=0.2$. The product space contains the four atoms

$$
\langle\text { go, win }\rangle,\langle\text { go, lose }\rangle,\langle\text { stay, win }\rangle \text {, and }\langle\text { stay, lose }\rangle \text {. }
$$

The latter two are causally impossible: If you do not go to the races, you can neither win nor lose. By the argument given above, it seems to follow that therefore, you go with probability 1 . This is absurd.

A way out of this quandary is to use the alternative to the product space construction that was introduced above: Consider only the consistent combinations of outcomes of $e_{1}$ and $e_{2}$ and take as the atoms the maximally consistent sets. Thus, we take the sample space of alternatives to be

$$
A=\left\{\left\{t_{1}, t_{2}^{\prime}\right\} \mid t_{2}^{\prime} \in\left[t_{2}\right]\right\} \cup\left\{\left\{t_{1}^{\prime}\right\} \mid t_{1}^{\prime} \in\left[t_{1}\right], t_{1}^{\prime} \neq t_{1}\right\} .
$$

Given finiteness, the Boolean algebra $F$ is again the usual set-theoretic algebra, and $\mu$ is uniquely specified by its value on the atoms. Each element of $A$ is a maximally detailed description of a consistent outcome involving the initials $e_{1}$ and (possibly) $e_{2}$, and each element of $F$ is a set of such consistent outcomes. $A$ then contains exactly the causal alternatives to $T$. In terms of the races example, we consider as atomic outcomes only the three (instead of four) maximally consistent sets (not pairs) of transitions,

$$
\{\text { go, win }\},\{\text { go, lose }\},\{\text { stay }\} \text {. }
$$

Note that while the set $\{$ go $\}$ is consistent, it is not maximally so and is thus not considered to be an atomic possibility. We may however identify that set with an element of $F$ : $\{$ go $\}$ says the same as $\{\{$ go, win $\},\{$ go, lose $\}\}-$ if you go, you either go and win, or you go and lose. A kind of marginal property is thus already built into our framework, since the probability of $\{\mathrm{go}\}$ evaluates as

$$
\mu(\{\text { go }\})=\mu(\{\{\text { go, } \operatorname{win}\},\{\text { go, lose }\}\})=\mu(\{\{\text { go, win }\}\})+\mu(\{\{\text { go }, \text { lose }\}\})
$$


by probability theory alone. We will spell this out by defining the notion of a representative below.

The framework sketched for our example easily generalizes to arbitrary finite causal probability structures, as will be shown in the following.

\subsection{General causal probabilities}

We start with a given consistent set $T$ of transitions, e.g., the set of causae causantes of a given transition $I \longmapsto O$. Since we only consider finite structures in this paper, we require that (i) $T$ be finite and that (ii) for each $t_{i} \in T$, $\left[t_{i}\right]$ be finite. ${ }^{8}$

The causal probability space $P_{T}=\left\langle A_{T}, F_{T}, \mu_{T}\right\rangle$ that is adequate to describe causal alternatives to $T$ is constructed as follows:

1. Let $\tilde{T}=\cup_{t_{i} \in T}\left[t_{i}\right]$, the set of all alternatives to the elements of $T$.

2. Let $A_{T}=\left\{T^{\prime} \mid T^{\prime} \subseteq \tilde{T}, T^{\prime}\right.$ maximally consistent $\}$.

3. Let $F_{T}$ be the set-theoretic Boolean algebra over $A_{T}$.

4. The measure $\mu_{T}$ may be any normalized measure on $F_{T}$.

5. The sought-for probability space is $P_{T}=\left\langle A_{T}, F_{T}, \mu_{T}\right\rangle$.

As one expects, the set $T$ of transitions turns out to be an element of $A_{T}$ since $T$ is consistent and by Lemma 3 (3a), no other element of $\tilde{T}$ can be consistently added to $T$. The given construction does not in any way single out $T$ from among the other elements of $A_{T}$. This is as it should be, since a probability space does not contain a designated element to stand for "the real outcome". Thus, the probability space $P_{T}$ can be constructed once the initials of all transitions in $T$ are known: it suffices to start from a given consistent set of point events $E$; the corresponding

\footnotetext{
${ }^{8}$ It turns out that while it is rather simple to generalize with respect to requirement (ii), using standard tools of measure theory (e.g., Borel sets), it is much harder to generalize with respect to (i). In fact, infinite $T$ creates difficulties for both considerations of probabilities and for the notion of "modal funny business". With respect to the latter, it turns out that Definition 5 is no longer adequate for guaranteeing "smooth combinatorics". In order to exclude combinatorially weird cases, it seems best to use the step from condition (3) to condition (1) in Lemma 3 as the mark of "no funny business". The whole area merits further study. - With respect to probabilities, if $T$ is infinite, i.e., involves infinitely many initials, then there may be an infinite SLR set of initials, and there may be an infinite chain of transitions in $T$. In the first case, the tools of standard probability theory for infinite product spaces (cylinder sets, zero-one laws) will apply. In the second case, the situation appears to be more challenging. Still that case is very interesting in view of the fact that, e.g., in the modal theory of agency, "busy choice sequences" are analyzed whose probabilistic equivalent exactly requires a probability theory for infinite chains of transitions. That question, too, certainly merits further study.
} 
probability space may then be denoted $P_{E}=\left\langle A_{E}, F_{E}, \mu_{E}\right\rangle$. Since this way of specifying causal probability structures can be generalized most easily, we will mainly be talking about probability space $P_{E}$ with a given set $E$ of initials. - It is even possible to build probability spaces from given inconsistent sets of point events; we will consider this generalization below.

In standard probability theory, it is customary to require that different measures in different probability spaces respect the marginal property, which was formulated for pairs as Definition 7 above. Since causal probability structures are not product spaces, we need some way of expressing the fact that we wish to consider the probability of "the same thing" in different probability spaces.

\subsubsection{Representation in different probability spaces}

Reconsider the races example from section 3.1.3. Going was in some sense an alternative, but it was not a maximally specific alternative and thus, not an element of $A_{T}$. Still there was a place for it in $F_{T}$, viz., as the set $\{\{$ go, win $\},\{$ go, lose $\}\}$. More generally, we may ask: Given a consistent set of basic transitions $S$, i.e., an element of the sample space $A_{E}$ of some causal probability space $P_{E}$, and a causal probability space $P_{E^{\prime}}$, how is $S$ represented in $P_{E^{\prime}}$ ? That question may be split in two: (i) When is $S$ representable in $P_{E^{\prime}}$ ? (ii) In case $S$ is representable, what is the representative? The answer to the first question is straightforward:

Definition 9 (Representability of $S$ in $P_{E^{\prime}}$ ) A consistent set $S \in A_{E}$ of basic transitions is representable in $P_{E^{\prime}}$ iff $E \subseteq E^{\prime}$.

When $S$ is representable, we have seen that we should not expect $S \in A_{E^{\prime}}$, since $A_{E^{\prime}}$ contains maximally consistent sets relative to $E^{\prime}$ only, and $S$ may not be maximal. Nor will we have $S \in F_{E^{\prime}}$, since $F_{E^{\prime}}$ is a set of (maximally consistent) sets of transitions. However, the following definition captures the required notion:

Definition 10 (Representative of $S$ in $P_{E^{\prime}}$ ) The representative of $S$ in $P_{E^{\prime}}, S_{E^{\prime}}$, is defined to be the set of all maximally consistent sets extending $S$ :

$$
S_{E^{\prime}}=\left\{T^{\prime} \in A_{E^{\prime}} \mid S \subseteq T^{\prime}\right\} .
$$

Thus we have $S_{E^{\prime}} \in F_{E^{\prime}}$. In order to visualize what is going on, it may be good to divide the definition into two steps, one downward and one upward, as follows:

1. $S^{\prime}=S \cup\left\{e^{\prime} \longmapsto \Pi_{e^{\prime}}\langle e\rangle \mid e \longmapsto H\right.$ minimal in $\left.S, e^{\prime}<e, e^{\prime} \in E^{\prime}\right\}$

In words: $S^{\prime}$ is derived from $S$ by "downward closure in $E^{\prime \prime}$, i.e., by adding all transitions below $S$ that are consistent with $S$ and that have initials in $E^{\prime}$. 
2. $S_{E^{\prime}}=\left\{T^{\prime} \in A_{E^{\prime}} \mid S^{\prime} \subseteq T^{\prime}\right\}$

In words: the representative of $S$ in $P_{E^{\prime}}$ is the set of all maximally consistent sets extending $S^{\prime}$. Since $S^{\prime}$ is downward closed in $E^{\prime}$, this step will only add elements on top of $S$.

The concept of a representative allows us to state, and prove, that the probability of a set of transitions is always smaller than or equal to the probability of its subsets:

Lemma 5 Let $S$ be representable in $P_{E^{\prime}}$, and let $S^{\prime} \subseteq S$. Then $\mu_{E^{\prime}}\left(S_{E^{\prime}}\right) \leq$ $\mu_{E^{\prime}}\left(S_{E^{\prime}}^{\prime}\right)$.

Proof: As $S^{\prime} \subseteq S, S^{\prime}$ is representable in $P_{E^{\prime}}$ as well. From the definition of $S_{E^{\prime}}$, one reads off $S_{E^{\prime}} \subseteq S_{E^{\prime}}^{\prime}$. The claim then follows from the additivity of $\mu_{E^{\prime}}$.

Representing elements of the event algebra So far, we have only considered the representation of consistent sets of transitions, i.e., elements of the sample space of some probability space. More generally, we may be interested in defining a representative for a set of consistent sets of transitions, e.g., an element of the event algebra of some probability space. The respective definitions are natural generalizations of the two definitions from above:

Definition 11 (Representability) A set $f \in F_{E}$ of sets of basic transitions is representable in $P_{E^{\prime}}$ iff $E \subseteq E^{\prime}$.

Thus, $f$ is representable in $P_{E^{\prime}}$ iff each $S \in f$ is representable in $P_{E^{\prime}}$.

Definition 12 (Representative of $f$ in $P_{E^{\prime}}$ ) The representative of $f$ in $P_{E^{\prime}}, f_{E^{\prime}}$, is defined to be the set of all representatives of elements of $f$ :

$$
f_{E^{\prime}}=\left\{S_{E^{\prime}} \mid S \in f\right\} .
$$

\subsubsection{Adapting the marginal property}

As in standard probability theory, we want to say that the probability of "something" is the same in all probability spaces representing the something. Taking the "something" to be a consistent set of basic transitions, one might think that we would like to have the following alternative marginal property:

(BAD-MP) If $S \in A_{E}$ is representable in $P_{E^{\prime}}$, then $\mu_{E}(S)=\mu_{E^{\prime}}\left(S_{E^{\prime}}\right)$.

In standard probability theory, this is a good way to describe the marginal property. In our framework, it will not do. Consider $S=\left\{e_{1} \longmapsto H_{1}\right\}$ and $E=\left\{e_{1}\right\}, E^{\prime}=$ $\left\{e_{0}, e_{1}\right\}$ with $e_{0}<e_{1}$. In this situation, the probability of $S=\left\{\left\{e_{1} \longmapsto H_{1}\right\}\right\}$ will 
normally be greater than the probability of $S_{E^{\prime}}=\left\{\left\{e_{0} \longmapsto \Pi_{e_{0}}\left\langle e_{1}\right\rangle, e_{1} \longmapsto H_{1}\right\}\right\}$, since in $P_{E^{\prime}}$, first $e_{0}$ has to have the right outcome and then $e_{1}$ has to have the right outcome, whereas in $P_{E}$, only $e_{1}$ has to have the right outcome. Standard probability theory with the product construction does not leave room for picturing more than one "layer" of initials, but the framework that is presented here does. In order to arrive at a correct formulation of the marginal property, we need to require that $P_{E^{\prime}}$ be an extension of $P_{E}$, which notion is defined as follows:

Definition 13 (Extension) A causal probability space $P_{E^{\prime}}$ is an extension of $P_{E}$ iff (i) $E \subseteq E^{\prime}$ and (ii) there is no $e^{\prime} \in E^{\prime}-E$ and $e \in E$ such that $e^{\prime}<e$.

In other words, $P_{E^{\prime}}$ is an extension of $P_{E}$ iff $E^{\prime}$ is a superset of $E$ and all the new elements of $E^{\prime}$ are maximal with respect to $E$. If $P_{E^{\prime}}$ extends $P_{E}$, the problematic situation described above cannot occur. Thus, the following is an adequate formulation of the marginal property for sets of basic transitions in our causal framework:

(MP) If $S \in A_{E}$ is representable in $P_{E^{\prime}}$ and if $P_{E^{\prime}}$ extends $P_{E}$, then $\mu_{E}(S)=$ $\mu_{E^{\prime}}\left(S_{E^{\prime}}\right)$.

We postulate that the marginal property should hold in Our world:

Postulate 2 The marginal property (MP) holds for all probability assignments.

Postulate 2 is already sufficient to ensure that an analogue of (MP) holds for elements of event algebras as well. We state this result as a Lemma:

Lemma 6 Let $f \in F_{E}$, and let $P_{E^{\prime}}$ extend $P_{E}$. Given $(M P)$, it follows that $\mu_{E}(f)=\mu_{E^{\prime}}\left(f_{E^{\prime}}\right)$.

Proof:

$$
\mu_{E}(f)=\sum_{S \in f} \mu_{E}(S)=\sum_{S \in f} \mu_{E^{\prime}}\left(S_{E^{\prime}}\right)=\mu_{E^{\prime}}\left(\left\{S_{E^{\prime}} \mid S \in f\right\}\right)=\mu_{E^{\prime}}\left(f_{E^{\prime}}\right) .
$$

\subsubsection{Markov property}

The motivation for the marginal property was purely structural: "the same thing" has to have the same probability, however represented. The causal structure only entered in spelling out what "representing the same thing" might mean. We now consider a property of probability spaces that is of causal origin and that accordingly does not have a direct counterpart in standard probability theory. We rely on 
the intuition that the probability of a transition is something like the probability of the outcome, given the initial. We will suggest that in some specific cases, this may be read as a conditional probability.

We first introduce the notion of layering:

Definition 14 Let $T$ be consistent. We say that $\mathrm{T}$ is layered iff there is a proper subset $T^{\prime}$ of $T$ such that for all $t^{\prime} \in T^{\prime}$ and for all $t \in T-T^{\prime}, t<t^{\prime}$. In this case, we say that $T^{\prime}$ forms an upper layer of $T$.

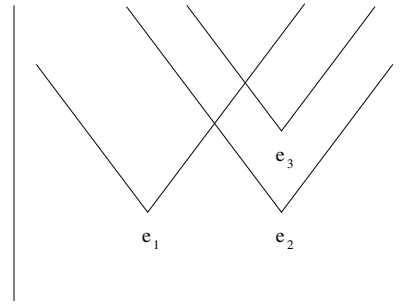

A

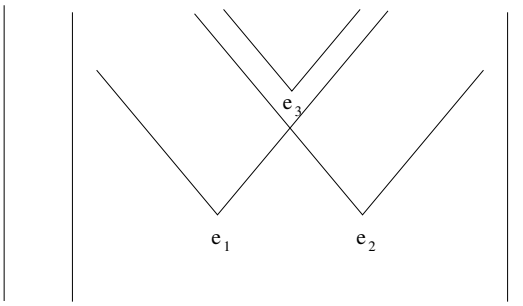

B

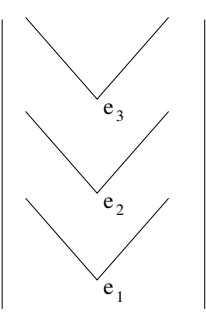

$\mathrm{C}$

Figure 2: A: not layered; B and C: layered

Not all $T$ are layered; consider Figure 2A, where $e_{2}<e_{3}$, but $e_{1} \operatorname{SLR} e_{2}$ and $e_{1}$ SLR $e_{3}$. If $T$ is layered, as in Figure $2 \mathrm{~B}$ and $\mathrm{C}$, an upper layer may be uniquely determined, as in (B), or it may not be unique, as in (C).

Transitions in an upper layer of layered $T$ all are located above all the other transitions. Thus there can be no causal influence from the transitions in $T-T^{\prime}$ on one of the outcomes of $T^{\prime}$ that was not already present when $T^{\prime}$ occurs. Given that $T^{\prime}$ occurs, the occurrence of $T-T^{\prime}$ has been accounted for completely. This observation motivates the following postulate:

Postulate 3 (Markov property) Let $T=R \cup S$ be consistent and layered, and let $R$ be an upper layer of $T$. Then all measures $\mu_{T}$ on $P_{T}$ satisfy

$$
\mu_{T}(T)=\mu_{R}(R) \cdot \mu_{S}(S)
$$

In case $\mu_{S}(S) \neq 0$, this may be phrased in terms of conditional probabilities:

$$
\mu_{R}(R)=\mu_{T}(T \mid S)=\mu_{T}\left(\{T\} \cap S_{T}\right) / \mu_{T}\left(S_{T}\right)=\mu_{T}(T) / \mu_{S}(S),
$$

where we have employed $T \in S_{T}$ (since $S \subseteq T$ ) and $\mu_{S}(S)=\mu_{T}\left(S_{T}\right)$ (by the marginal property). 
Note that we are here dealing with an ontologically motivated Markov condition that applies to single cases. In connection with graphical models and Bayesian Networks, one assumes a so-called "causal Markov condition" that applies to "variables". We will give some brief remarks on the interrelation between the framework presented here and the framework of Bayesian Networks at the end of section 3.2.6 below.

\subsubsection{Causal probability spaces from inconsistent initials}

Technically, it is unproblematic to extend the probability space construction described at the beginning of section 3.2 to any set $E$ of initials:

- Form the set $\tilde{T}=\cup_{e \in E}[e]$.

- Use the set of all maximally consistent subsets of $\tilde{T}$ as sample space $A_{E}$.

- Form the event algebra $F_{E}$; in the finite case that is assumed here for simplicity, use the set-theoretic algebra of subsets of $A_{E}$.

- Define some suitable measure $\mu_{E}$ on $F_{E}$.

- Set the probability space $P_{E}$ to be $\left\langle A_{E}, F_{E}, \mu_{E}\right\rangle$.

For this construction to be well-defined it is not necessary that $E$ be consistent. E.g., in the case of $E=\left\{e_{1}, e_{2}\right\}$ with incompatible $e_{1}$ and $e_{2}$, assuming two outcomes $+_{1},-{ }_{1}$ and $+_{2},-{ }_{2}$ for simplicity, $A_{E}$ contains only singletons:

$$
A_{E}=\left\{\left\{e_{1} \longmapsto+_{1}\right\},\left\{e_{1} \longmapsto-{ }_{1}\right\},\left\{e_{2} \longmapsto+_{2}\right\},\left\{e_{2} \longmapsto-{ }_{2}\right\}\right\} .
$$

The crucial question is: under which circumstances can we give a coherent causal reading to the space $P_{E}$ ? The guiding idea in constructing probability spaces was that $A_{E}$ should describe alternatives. In the example, this reading appears questionable: in which sense might the occurrence of $e_{1} \longmapsto+_{1}$ be an alternative to the occurrence of $e_{2} \longmapsto+_{2}$ ? A minimal sense of "alternative" might be defended: They cannot both occur, so they are alternatives. But by looking for a causal reading of probability spaces, we also want a causal sense of "alternative", and that sense is not forthcoming in the example: No connection between the alternatives is present. Things would be different if $E$ contained a third element $e$ such that $e<e_{1}, e<e_{2}$, for then, $e$ would provide the sought-for causal link, shared by all alternatives.

In the general case, we want to say that $A_{E}$ is a set of causal alternatives if there is such a causal link in the form of initials that are shared by all the alternatives. Since $A_{E}$ consists of maximally consistent sets of transitions, the minima of $E$ will 
be common initials of all alternatives in $A_{E}$ exactly if they are consistent. We thus give the following definition of when a probability space is causally interpretable as a space of alternatives: ${ }^{9}$

Definition 15 (Causal probability space) Let $E$ be a finite set of finitely splitting point events. The probability space $P_{E}$, defined as above, is a causal probability space iff the minimal elements of $E$ are consistent.

Given a causal probability space $P_{E}$, it will do no harm to add proper maxima to $E$ - the resulting probability space will again be causal, since its minimal elements stay the same. We call the procedure of adding proper maxima "fine graining". For the idea, refer back to the races example from section 3.1.3. There, $E=\left\{e_{1}, e_{2}\right\}$, and there was one minimal initial, $e_{1}$ (deciding what to do), with outcomes go/stay. In the outcome "go", there was a second initial, $e_{2}$ (betting), with outcomes win/lose. By our definition, the corresponding probability space $P_{E}$ is causal. That feature will be preserved if we give a more fine-grained description of the "stay" outcome of $e_{1}$ by adding a new initial $e_{3}$ in the "stay" outcome above $e_{1}$. E.g., $e_{3}$ might have outcomes read/cook. With $E^{\prime}=\left\{e_{1}, e_{2}, e_{3}\right\}, P_{E^{\prime}}$ clearly describes causal alternatives: you either stay and read, or you stay and cook, or you go and win, or you go and lose. Given one alternative, you can tell a causal story of how another alternative might have occurred. Note that $P_{E^{\prime}}$ cannot be derived from any consistent set of transitions directly, but only via fine graining. Our formal definition runs as follows:

Definition 16 (Fine graining) Let $E$ and $E^{\prime}$ be finite sets of finitely splitting point events. The probability space $P_{E^{\prime}}$ is a fine-grained version of $P_{E}$ iff (i) $E \subseteq E^{\prime}$, (ii) for all $e^{\prime} \in E^{\prime}-E$ there is $e \in E$ such that $e<e^{\prime}$, and (iii) for all $S \in A_{E}$, the measure is preserved: $\mu_{E}(S)=\mu_{E^{\prime}}\left(S_{E^{\prime}}\right)$.

Thus, fine graining means adding new initials that are not new minima, while preserving the measure as far as possible. We can now formulate a Lemma that connects the notion of a causal probability space with the notion of fine graining.

Lemma 7 Let $E$ be a set of point events. The following conditions are equivalent:

1. $P_{E}$ is a causal probability space (i.e., the minima of $E$ are consistent).

2. The minima of $E$ are pairwise space-like related.

\footnotetext{
${ }^{9}$ The definition is limited to the finite case in view of the general finiteness requirement in this paper.
} 
3. $P_{E}$ is a fine-grained version of a causal probability space $P_{T}$, where $T$ is a consistent set of transitions.

Proof: $1 \Rightarrow 2$ : Since the set of minima is consistent, there is a history $h$ containing them all. Two minima $e_{1}$ and $e_{2}, e_{1} \neq e_{2}$, cannot be comparable (for then one would not be minimal), so (as witnessed by $h$ ) they are space-like related.

$2 \Rightarrow 3$ : Let $M$ be the set of minima of $E$. For each $e_{i} \in M$, select an outcome $H_{i} \in \Pi_{e_{i}}$, and let $T=\left\{e_{i} \longmapsto H_{i}\right\}$. By Lemma 3 (3d), $T$ is consistent. Then $P_{E}$ is derived by fine graining from $P_{T}$ : (i) $M \subseteq E$ and (ii) $E-M$ contains just the non-minimal elements of $E$. The measure can be adjusted as necessary.

$3 \Rightarrow 1$ : Let $P_{E}$ be derived from $P_{T}$ by fine graining, and let $M$ be the set of minima from among the initials of $T$. By consistency of $T, M$ is consistent, and by the definition of fine graining, $M$ is also the set of minima of $E$.

\subsubsection{Random variables and correlations}

In standard probability theory, a random variable $X$ is a function defined on the sample space $A$ of a probability space $P$. Usually, but not necessarily, the values of $X$ will be real numbers. E.g., with the sample space $A$ being the outcomes of a roll of a die, $X$ might be equal to one for the odd outcomes and equal to zero for the even ones. The probability of a value $x$ of the random variable $X$ is written $\operatorname{pr}(X=x)$. For a fair die, in the example case we would thus have $\operatorname{pr}(X=1)=1 / 2$.

Random variables may map more than one element of the sample space to the same value, as in the example. The most fine-grained random variables preserve the structure of the sample space via an isomorphism, e.g., $X(a)=a$. For such random variables, we may identify $\operatorname{pr}(X=x)$ with $\mu(x)$. This identification is unproblematic in the finite case considered here. Random variables are required to give a definition of correlation and (probabilistic) independence:

Definition 17 (Independence and correlations) A family $X_{1}, \ldots, X_{n}$ of random variables defined on a probability space $P$ is called independent iff for any $n$-tuple $\left\langle x_{1}, \ldots, x_{n}\right\rangle$ of respective outcomes,

$$
\operatorname{pr}\left(X_{1}=x_{1} \wedge \ldots \wedge X_{n}=x_{n}\right)=\operatorname{pr}\left(X_{1}=x_{1}\right) \cdot \ldots \cdot \operatorname{pr}\left(X_{n}=x_{n}\right) .
$$

If the family $X_{1}, \ldots, X_{n}$ is not independent, it is called correlated. Thus, a correlation is of the form

$$
\operatorname{pr}\left(X_{1}=x_{1} \wedge \ldots \wedge X_{n}=x_{n}\right) \neq \operatorname{pr}\left(X_{1}=x_{1}\right) \cdot \ldots \cdot \operatorname{pr}\left(X_{n}=x_{n}\right) .
$$


Note that pairwise independence does not guarantee independence. - It is straightforward to transfer these notions to causal probability structures: A random variable $X_{E}$ is again taken to be a function defined on the sample space $A_{E}$ of a probability space $P_{E}$. Basic random variables are defined on the sample space $A_{\{t\}}$ generated by a single basic transition $t=e \longmapsto H$.

As an example, consider rolling a fair die again, as described by a probability space $P_{E}(E=\{e\})$. The random variable $X$ will be as defined above. Let $Y$ be defined to have the value one for outcome 2 and value zero otherwise. It turns out that $X$ and $Y$ are correlated, since, e.g.,

$$
\operatorname{pr}(X=1 \wedge Y=1)=0 \neq \operatorname{pr}(X=1) \cdot \operatorname{pr}(Y=1)=(1 / 2) \cdot(1 / 6) .
$$

Such trivial correlations must be distinguished from empirically challenging correlations, for which the notion of screening-off is important. Before we move to that discussion, we give a definition of independence and correlations for causal probability spaces, which is based on using the most fine-grained random variables:

Definition 18 Let $P_{E}$ be a finite causal probability space. $P_{E}$ is called (probabilistically) independent iff for any $T=\left\{t_{1}, \ldots, t_{n}\right\} \in A_{E}$,

$$
\mu_{E}(T)=\prod_{t_{i} \in T} \mu_{\left\{t_{i}\right\}}\left(t_{i}\right)
$$

$P_{E}$ is called correlated iff it is not independent.

Thus, we have at our hands two different concepts of correlations: One is about random variables defined on $P_{E}$, while the other is about $P_{E}$ itself. The die example already shows that on an uncorrelated $P_{E}$, one can define correlated random variables. In many empirically important cases, such correlations among random variables may be explained by uncorrelated $P_{E}$, invoking the notion of (probabilistic) screening-off.

\subsubsection{Screening-off}

One of the most important links between causality and probability is via the concept of screening-off. Roughly, screening-off means that if there are correlations among causally unconnected variables, then in the common past of these variables there is an event that screens off the correlations, i.e., conditional on which there are no correlations any more. Consider the notorious barometer example: A falling barometer is correlated with rain, even though neither does the rain cause the barometer to fall, nor does the falling barometer cause the rain. In this situation, we can point to a temporally prior common cause of the correlations: Low 
atmospheric pressure causes both the rain and the barometer's falling; conditional on low atmospheric pressure, the two variables are uncorrelated.

This story is good as far as it goes, and it captures a methodologically important point that may drive empirical research: Explain correlations through the common past. ${ }^{10}$ Since we wish to give a reading of screening-off in the framework of this paper, we need to be more explicit with respect to a number of key notions:

1. What are the variables that may be correlated?

2. For which correlations do we expect screening-off?

3. Which notion of location for variables is appropriate?

4. What does the screening-off principle look like? How can the requirement of causal priority and the concept of conditioning on a common cause be expressed?

(1) It seems natural to take "variables" to be random variables defined on a causal probability space $P_{E}$. Thus, we will be dealing with correlations in the sense of Definition 17.

(2) As a famous slogan would have it, correlations cry out for explanation. That may be so, but certainly not all correlations cry for explanations of the screeningoff variety. That is already obvious from the die example, where the random variables were just defined so as to yield correlations. Furthermore, any sort of causal influence of one variable on another will make correlations relatively uninteresting, too. E.g., in the races example from section 3.1.3, going is correlated with winning, but this is just so because in order to win, one has to go and bet in the first place. Thus, interesting correlations are among "causally separated" variables, which must somehow be expressed via the concept of space-like separation, bringing us to the next point.

(3) Random variables do not have locations. Still, for a random variable $X$ defined in $P_{E}$, some points $e \in E$ may be important for determining the value of $X$, while others may be unimportant. The maximal elements of $E$ play a special role here, since the outcomes of the maximal elements determine an element of the sample space (the domain of $X$ ) uniquely. Letting $E_{M}$ be the set of maximal elements of $E$, we thus define the set $V_{X}$ of value-determining points for $X$ to be

$V_{X}=\left\{e \in E_{M} \mid \exists t, t^{\prime} \in[e] \exists S \in A_{E}\left(t \in S \wedge X(S) \neq X\left((S-\{t\}) \cup\left\{t^{\prime}\right\}\right)\right)\right\}$.

\footnotetext{
${ }^{10}$ In fact, the first overt application of a screening-off principle, by Reichenbach (1956), was in trying to define the direction of time.
} 
In words, a maximal point $e$ of $E$ is value-determining for $X$ if the outcome of $e$ can make a difference as to the value of $X$.

We may now define when two random variables defined in $P_{E}$ are causally separated:

Definition 19 (Causal separation of random variables) The random variables $X$ and $Y$ defined in $P_{E}$ are causally separated iff their respective value-determining points are either space-like separated or incompatible, i.e., iff

$$
\forall e_{X} \in V_{X} \forall e_{Y} \in V_{Y}\left(e_{X} \operatorname{SLR} e_{y} \vee e_{X}, e_{Y} \text { incompatible }\right)
$$

Thus, we will be looking for screening-off for causally separated variables only. (This excludes both the die case and the races example.)

(4) Let $X$ and $Y$ be two causally separated, correlated random variables defined in a causal probability space $P_{E}$. If $P_{E}$ itself is correlated (according to Definition 18), then correlations among variables are to be expected anyhow. However, in the absence of correlations in $P_{E}$, a screening-off principle holds. We state this as a Theorem:

\section{Theorem 1 (Screening-off principle)}

Let $X$ and $Y$ be two causally separated, correlated random variables defined in a causal probability space $P_{E}$, and let $E^{\prime}=E-E_{M}$ be the set of non-maximal elements in $E$. Then either (i) $P_{E}$ is correlated or (ii) $P_{E}$ is uncorrelated, and any $S^{\prime} \in A_{E^{\prime}}$ screens off $X$ from $Y$ : For any $S^{\prime} \in A_{E^{\prime}}$, any $x \in \operatorname{ran}(X)$ and any $y \in \operatorname{ran}(Y)$,

$$
\operatorname{pr}\left(X=x \wedge Y=y \mid S^{\prime}\right)=\operatorname{pr}\left(X=x \mid S^{\prime}\right) \cdot \operatorname{pr}\left(Y=y \mid S^{\prime}\right) .
$$

Proof: We need to consider (ii) only. For simplicity's sake we assume that $V_{X} \cup$ $V_{Y}=E_{M}$, i.e., all maximal points in $E$ are relevant for $X$ or for $Y$. (Otherwise, the unnecessary points may be deleted.) We write $S_{x}\left(S^{\prime}\right)$ for the set $\left\{S \in A_{E} \mid\right.$ $\left.S^{\prime} \subseteq S, X(S)=x\right\}, S_{y}$ analogously, and $S_{x y}$ for $\left\{S \in A_{E} \mid S^{\prime} \subseteq S, X(S)=\right.$ $x \wedge Y(S)=y$. Thus, e.g., $S_{x}\left(S^{\prime}\right)$ pools together all ways of continuing $S^{\prime}$ such as to yield value $x$ for $X$. Then we have

$$
\begin{aligned}
\operatorname{pr}\left(X=x \wedge Y=y \mid S^{\prime}\right) & =\mu_{E}\left(\left\{S \in S_{x y}\left(S^{\prime}\right)\right\}\right) / \mu_{E^{\prime}}\left(S^{\prime}\right) \\
& =\sum_{S \in S_{x y}\left(S^{\prime}\right)} \mu_{E}(S) / \mu_{E^{\prime}}\left(S^{\prime}\right) \\
& =\sum_{S \in S_{x y}\left(S^{\prime}\right)} \prod_{t \in S-S^{\prime}} \mu_{\{t\}}(t) .
\end{aligned}
$$


The fraction may be replaced by the product in the last step since $P_{E}$ is uncorrelated and thus, both $\mu_{E}(S)$ and $\mu_{E^{\prime}}\left(S^{\prime}\right)$ factor: e.g., $\mu_{E}(S)=\prod_{t \in S} \mu_{\{t\}}(t)$. We now write $T_{x}\left(S^{\prime}\right)$ for the set of sets of transitions $T$ with initials from $V_{X}$ for which there is $S \in A_{E}, S^{\prime} \subseteq S, T \subseteq S$, with $X(S)=x$. Thus, $T_{x}\left(S^{\prime}\right)$ pools together all possible ways for the variable $X$ to have value $x$, given that $S^{\prime}$ occurred. (The outcomes of $e \in V_{Y}$ do not change the value of $X$.) We thus get

$$
\begin{aligned}
\operatorname{pr}\left(X=x \mid S^{\prime}\right) & =\mu_{E}\left(\left\{S \in S_{x}\left(S^{\prime}\right)\right\}\right) / \mu_{E^{\prime}}\left(S^{\prime}\right) \\
& =\sum_{S \in S_{x}\left(S^{\prime}\right)} \mu_{E}(S) / \mu_{E^{\prime}}\left(S^{\prime}\right) \\
& =\sum_{S \in S_{x}\left(S^{\prime}\right)} \prod_{t \in S-S^{\prime}} \mu_{\{t\}}(t) \\
& =\sum_{T \in T_{x}\left(S^{\prime}\right)} \prod_{t \in T} \mu_{\{t\}}(t) .
\end{aligned}
$$

The last step is licensed since for $e \notin V_{X}$, all outcomes of $e$ will lead to the same value of $X$ by definition, so that the sum is over all outcomes of $e$, yielding probability one and thus canceling $e$ from the sum.

We finally have

$$
\begin{gathered}
\operatorname{pr}\left(X=x \mid S^{\prime}\right) \cdot \operatorname{pr}\left(Y=y \mid S^{\prime}\right)= \\
\left(\sum_{T \in T_{x}\left(S^{\prime}\right)} \prod_{t \in T} \mu_{\{t\}}(t)\right) \cdot\left(\sum_{T^{\prime} \in T_{y}\left(S^{\prime}\right)} \prod_{t^{\prime} \in T^{\prime}} \mu_{\left\{t^{\prime}\right\}}\left(t^{\prime}\right)\right)= \\
\sum_{T \in T_{x}\left(S^{\prime}\right)} \sum_{T^{\prime} \in T_{y}\left(S^{\prime}\right)} \prod_{t \in T} \mu_{\{t\}}(t) \cdot \prod_{t^{\prime} \in T^{\prime}} \mu_{\left\{t^{\prime}\right\}}\left(t^{\prime}\right)= \\
\sum_{S \in S_{x y}\left(S^{\prime}\right)} \prod_{t \in S-S^{\prime}} \mu_{\{t\}}(t)=\operatorname{pr}\left(X=x \wedge Y=y \mid S^{\prime}\right) .
\end{gathered}
$$

In the last step, we have employed the assumption that $V_{X}$ and $V_{Y}$ together make up the set of maxima of $E$, so that any continuation of $S^{\prime}$ in $A_{E}$ has "new" initials either from $V_{X}$ or from $V_{Y}$.

Thus, our framework allows to capture in a mathematically precise way a notion of "prior screening-off" for correlated random variables: If variables $X$ and $Y$ are causally separated and the causal probability space $P_{E}$ on which $X$ and $Y$ are defined is uncorrelated, then the non-maximal elements of $E$ collectively act as screeners-off. Even though random variables do not have a space-time location, the Theorem captures the notion of prior screening-off, since the value of $X$ and 
$Y$ is determined by the maximal elements of $E$, which are causally later than the screeners-off.

Reichenbach seems to have thought that screening-off was a universal principle. Our Theorem, however, allows for a failure of screening-off, viz., if $P_{E}$ itself is correlated. Correlations in $P_{E}$ may in turn have two very different reasons: (i) It may be that $P_{E}$ is inadequate to describe the situation at hand, since it contains correlations where a more careful analysis, extending $P_{E}$, would find none. $P_{E}$ may thus be thought of as a "surface model" for the phenomena. In an extended model (made up from copies of $P_{E}$ after identifying prior screeners-off), there will be no correlations. (ii) It may be that $P_{E}$ is the right model, and still there are correlations.

A universal principle of screening-off will not rule out possibility (i). In fact, the principle of screening-off, read as a piece of scientific methodology, urges us to replace situations of type (i) with a more satisfactory, uncorrelated extended model. However, universal screening-off speaks against possibility (ii). It is an empirical question whether a situation of type (ii) actually occurs. Here, the Bell inequalities together with empirical results of quantum correlation experiments strongly suggest a positive answer: In nature, there are correlations among causally separated variables for which there is no screener-off. ${ }^{11}$ In the light of the strangeness of these correlations, it seems appropriate to call correlations in $P_{E}$ "probabilistic funny business". Thus, we define:

Definition 20 A causal probability space $P_{E}$ exhibits probabilistic funny business iff it is correlated according to Definition 18.

A short comment on the theory of Bayesian Networks may be in order. While this paper is about deducing probabilistic consequences from causal relations, it is often important to deduce causal relations from statistical data. Since the 1980s, a number of methods have been developed for elucidating that direction of the problem. The framework of Bayesian Networks (Pearl, 2000; Jensen, 2001) has been especially prominent in this respect, and many important applications have been based on that framework. The present framework may be seen as giving a spatiotemporally and mathematically precise reading of the "causal variables" of the Bayesian Network theory in terms of random variables defined on causal probability spaces. It seems plausible to assume that the further development of the present framework

\footnotetext{
${ }^{11}$ The Bell inequalities were derived by John Bell after Reichenbach's death; cf. Bell (1987) for the original papers dating from 1964 onward. Decisive experiments have been conducted since the 1980s; cf., e.g., Aspect et al. (1982). - A more thorough analysis of the quantum mechanical case will have to be deferred to a future paper.
} 
will shed light on fundamental questions in the theory of Bayesian Networks, such as the question of the status of the "causal Markov condition" assumed there. ${ }^{12}$

In the following section we will put many of our notions to work.

\subsection{Application: Probability of suprema of a chain}

As an application of our framework, we consider the following problem:

Let $I$ be an initial chain and Sup $:=\left\{\sup _{h}(I) \mid h \in H_{[I]}\right\}$ the family of its suprema. This is a kind of chance set-up that Belnap (1992) has called "indeterminism without choice": the transition from $I$ to its suprema is generally indeterministic, but no choice in $I$ determines the outcome. With respect to this set-up, two questions arise:

1. In which probability space may we describe all the transitions $I \longmapsto p, p \in$ Sup, as causal alternatives?

2. What will be the sum of the probabilities of all these alternative transitions? Will our framework yield the expected answer,

$$
\sum_{p \in \text { Sup }} \mu(I \longmapsto p)=1 ?
$$

It turns out that we need a further Postulate to deal with this problem. Appendix A shows that that Postulate, due to Weiner (1997), is not a consequence of the other Postulates of branching space-times.

Postulate 4 (Weiner) Suprema retain their order across histories: If I and $J$ are two initial chains and $h_{1}, h_{2}$ two histories both containing both $I$ and $J$, then (i) $\sup _{h_{1}}(I)=\sup _{h_{1}}(J)$ iff $\sup _{h_{2}}(I)=\sup _{h_{2}}(J)$, (ii) $\sup _{h_{1}}(I)<\sup _{h_{1}}(J)$ iff $\sup _{h_{2}}(I)<\sup _{h_{2}}(J)$, and (iii) $\sup _{h_{1}}(I)>\sup _{h_{1}}(J)$ iff $\sup _{h_{2}}(I)>\sup _{h_{2}}(J)$.

Given this Postulate, the answer to the two questions is: (1) There is a natural choice for the sought-for probability space. (2) The probabilities in that space do sum to one. We formulate this as a Theorem. At present, since the considerations of probability spaces were limited to the finite case, we will have to assume finiteness here, too.

\footnotetext{
${ }^{12} \mathrm{~A}$ further interesting link between the two frameworks is given by the problem of applying the Bayesian Networks approach to single cases; cf. Pearl (2000, chap. 10) on the problem of "the actual cause".
} 


\section{Theorem 2}

Let $I$ be an initial chain, and let $S u p:=\left\{\sup _{h}(I) \mid h \in H_{[I]}\right\}$ be the family of its suprema. Let $C C=\cup_{p \in S u p} c c(I \longmapsto p)$. Then, assuming that $C C$ is finite and with finitely splitting initials,

1. $P_{C C}$ is a causal probability space,

2. $A_{C C}=\{c c(I \longmapsto p) \mid p \in S u p\}$, and

3. for any measure $\mu_{C C}$ on $F_{C C}, \sum_{p \in S u p} \mu_{C C}(I \longmapsto p)=1$.

Proof: Let $I$ and $S u p$ be given, and let $P C L=\cup_{p \in \operatorname{Sup}} \operatorname{pcl}(I \longmapsto p), C C=$ $\cup_{p \in S u p} c c(I \longmapsto p)$. We prove the three claims in turn, spending most of our labor on (2). First we need to prove a Lemma:

Lemma 8 Let $e_{i}$ be minimal in PCL, and let $p_{j} \in$ Sup. Then $e_{i}<p_{j}$.

Proof: As $e_{i} \in P C L$, there is $p_{i} \in S u p$ s.t. $e_{i}<p_{i}$. Assume that $e_{i} \nless p_{j}$. In that case, it follows from Postulate 4 that no history can contain both $e_{i}$ and $p_{j}$ (use $\left\{e_{i}\right\}$ and $I$ as initials and two histories both containing $e_{i}$ and one containing $p_{i}$, the other $p_{j}$ ). Thus, on the assumption that $e_{i} \nless p_{j}$, for a history $h_{i}$ containing $e_{i}$ and a history $h_{j}$ containing $p_{j}$, the prior choice principle gives a splitting point $e \in h_{i} \cap h_{j}$ s.t. $e<e_{i}$. But then $e<p_{i}$, and as $p_{j} \in h_{j}, h_{j} \in H_{[I]}$, and $h_{j} \perp_{e} H_{\left\langle p_{i}\right\rangle}$ by transitivity of undividedness. Thus, $e \in \operatorname{pcl}\left(I \longmapsto p_{i}\right)$, contradicting the minimality of $e_{i}$.

We now proceed to prove the Theorem.

(1) If $P C L$ has only one minimum, there is nothing to show. So let $e_{1}$ and $e_{2}$, $e_{1} \neq e_{2}$, be minima of PCL. Let $p \in S u p$. By Lemma 8, $e_{1}<p$ and $e_{2}<p$, so there is a history containing both. Since $e_{1}$ and $e_{2}$, being minima, must be incomparable, it follows that they are space-like related. The claim then follows by Lemma 7 (2).

(2) " $\subseteq$ ": For each transition $I \longmapsto p, p \in S u p, c c(I \longmapsto p)$ is a maximally consistent subset of $C C$.

Let $p \in S u p$, and let $T=c c(I \longmapsto p)$. By Lemma $1, T$ is consistent, and $T \subset C C$ by construction of $C C$. We need to show that $T$ is maximally consistent in $C C$. So let $t^{\prime}=e^{\prime} \longmapsto H^{\prime} \in C C-T$, and let $T^{\prime}=T \cup\left\{t^{\prime}\right\}$. Since $t^{\prime} \in C C$, $t^{\prime} \in c c\left(I \longmapsto p^{\prime}\right)$ for some $p^{\prime} \in S u p, p^{\prime} \neq p$, and $H^{\prime}=\Pi_{e^{\prime}}\left\langle p^{\prime}\right\rangle$. We will show that $T^{\prime}$ is inconsistent.

We first show that on the assumption that $T^{\prime}$ is consistent, $e^{\prime}<p$ : Assume that there is no history containing both $e^{\prime}$ and $p$. Then there must be a splitting point $e^{\prime \prime}<e^{\prime}$, and as in the proof of Lemma 8, we get $e^{\prime \prime} \in \operatorname{pcl}(I \longmapsto p)$, so 
$e^{\prime \prime} \longmapsto \Pi_{e^{\prime \prime}}\langle p\rangle \in T$. But as $e^{\prime \prime}$ is a splitting point for $H_{\left(e^{\prime}\right)}$ and $H_{\langle p\rangle)}, \Pi_{e^{\prime \prime}}\langle p\rangle \cap$ $H_{\left(e^{\prime}\right)}=\emptyset$, so that $T^{\prime}$ would be inconsistent. Thus there is a history containing both $e^{\prime}$ and $p$, and by Postulate $4, e^{\prime}<p$. - Now again as in the proof of Lemma 8, $e^{\prime} \in \operatorname{pcl}(I \longmapsto p)$, and thus either $t^{\prime} \in T$, contradicting our assumption $t^{\prime} \notin T$, or $T^{\prime}$ is inconsistent by Lemma 3 (3).

(2) "卫": Each maximally consistent subset of $C C$ is the set of causae causantes of a transition $I \longmapsto p, p \in S u p$. We show (a) each consistent subset of $C C$ determines a supremum of $I$, (b) each maximally consistent subset of $C C$ does so uniquely, and (c) a maximally consistent subset of $C C$ is in fact equal to the corresponding set of causae causantes.

(a) Let $S$ be a consistent subset of $C C$, let $S_{m}$ be the set of transitions maximal in $S$ (which exists by finiteness). We show that there is a history in $\cap_{t_{i} \in S_{m}} H_{i}$ that contains $I$ :

(a.i) For $t_{i} \in S_{m}$, all $e_{i}$ are pairwise space-like related (by maximality) and belong to one history (Lemma 3 (2)).

(a.ii) Also, for each $e_{i}$ there is a point $e \in I$ s.t. for all $e^{\prime} \in I$, if $e^{\prime} \geq e$, then $e^{\prime} \operatorname{SLR} e_{i}:(\alpha) e_{i}$ is a past cause-like locus for some transition $I \longmapsto p, p \in S u p$. So $e_{i}<p$, and there is a history $h$ such that $e_{i}$ and $I$ belong to $h$ and for $h_{p} \in H_{\langle p\rangle}$, $h \perp_{e_{i}} h_{p}$. ( $\beta$ ) For all $e \in I, e_{i} \nless e$. Assume otherwise. Then $e \in h$ (since $I \subseteq h$ ). Select $h_{p} \in H_{\langle p\rangle}$. Since $I<p, e<p$, so $e \in h_{p}$. But $e_{i}<e$ now gives $h \equiv_{e_{i}} h_{p}$, contradicting $h_{p} \in H_{\langle p\rangle}$. $(\gamma)$ There is $e \in I$ such that $e_{i} \geq e$. Otherwise, $e_{i}$ is an upper bound for $I$, and $e_{i} \in h$, so $e_{i} \geq p^{\prime}=\sup _{h}(I)$. But for $p \in S u p$ we also had $p<e_{i}$. By Postulate 4 (using $I$ and $\left\{e_{i}\right\}$ ), this is impossible. So $e$ SLR $e_{i}$. $(\delta)$ For $e^{\prime} \in I$, if $e<e^{\prime}$, then also $e^{\prime} \operatorname{SLR} e_{i}$ - otherwise, $e<e^{\prime} \leq e_{i}$, contradicting $(\gamma)$

(a.iii) Since $S_{m}$ is finite, the intersection of the final sections of $I$ constructed in step (a.ii) is nonempty, call it $I^{\prime}$. We have established $\left\{e_{i} \mid t_{i} \in S_{m}\right\}$ SLR $I^{\prime}$.

(a.iv) At this point we may use Postulate 1 for the primary outcome $\cap_{t_{i} \in S_{m}} H_{i}$ of the set $\left\{e_{i} \mid t_{i} \in S_{m}\right\}$ and for some primary outcome of $I^{\prime}$, this will give us a joint outcome and thus a history $h$ containing $I^{\prime}$ (and thus, $I$ ) and contained in all the outcomes $H_{i}$ of $t_{i} \in S_{m}$. - Since $S_{m}$ was maximal, $h \in \cap_{t_{i} \in S} H_{i}$. So $S$ determines the transition $I \longmapsto \sup _{h}(I)$.

(b) Now let $S$ be maximally consistent, and let $h_{1}, h_{2} \in \cap_{t_{i} \in S} H_{i}$ with $I \subseteq h_{1}$ and $I \subseteq h_{2}$. Set $p_{i}=\sup _{h_{i}}(I), i=1,2$. We want to show $p_{1}=p_{2}$. Assume $p_{1} \neq p_{2}$. (i) By the prior choice principle, since $p_{1} \in h_{1}-h_{2}$, there is a choice point $e<p_{1}$ such that $h_{1} \perp_{e} h_{2}$ (i.e., $e$ is maximal in $h_{1} \cap h_{2}$ ). By transitivity of undividedness, $h_{1} \perp_{e} H_{\left\langle p_{2}\right\rangle}$. So $e \in \operatorname{pcl}\left(I \longmapsto p_{2}\right)$. (ii) The transition $t=$ $e \longmapsto \Pi_{e}\left\langle p_{2}\right\rangle$ is a causa causans for $p_{2}$, so $t \in C C$. But $t \notin S$, since $t$ rules out $h_{1}\left(h_{1} \notin \Pi_{e}\left\langle p_{2}\right\rangle\right)$, and we had $h_{1} \in \cap_{t_{i} \in S} H_{i}$. (iii) Since also $h_{2} \in \cap_{t_{i} \in S} H_{i}$ and 
$h_{2} \in \Pi_{e}\left\langle p_{2}\right\rangle$, the set $S \cup\{t\}$ is consistent. This contradicts the assumption that $S$ is maximally consistent in $C C$.

(c) Let $S \subseteq C C$ be maximally consistent, and let $p \in S u p$ be the uniquely determined supremum of $I$ for which $H_{\langle p\rangle} \cap \bigcap_{t_{i} \in S} H_{i} \neq \emptyset$. We now show that $S=c c(I \longmapsto p)$.

(c) "ᄃ": Let $t_{i}=e_{i} \longmapsto H_{i} \in S$. We have $e_{i}<p$, and as $e_{i} \in P C L$, we also have $e_{i} \in \operatorname{pcl}(I \longmapsto p)$, and as $H_{i}=\Pi_{e_{i}}\langle p\rangle$, in fact $t_{i} \in c c(I \longmapsto p)$.

(c) "卫": Let $t_{i}=e_{i} \longmapsto H_{i} \notin S$. Then $S \cup\left\{t_{i}\right\}$ must be inconsistent (since $S$ is maximally consistent), and thus it cannot be that there is $h_{p} \in H_{\langle p\rangle} \cap H_{i}$ (else that history would witness consistency). But then $t_{i} \notin c c(I \longmapsto p)$.

(3) The last part of the proof is simple: Since $A_{C C}=\{c c(I \longmapsto p) \mid p \in S u p\}$, the sum in question turns out to be the measure of the unit element $\mathbf{1}_{C C}$, equal to one for any measure $\mu_{C C}$. In detail:

$$
\begin{aligned}
\sum_{p \in \text { Sup }} \mu(I \longmapsto p) & =\sum_{p \in \text { Sup }} \mu_{C C}(c c(I \longmapsto p))=\sum_{T \in A_{C C}} \mu_{C C}(T) \\
& =\mu_{C C}\left(\cup_{T \in A_{C C}} T\right) \\
& =\mu_{C C}\left(\mathbf{1}_{C C}\right)=1 .
\end{aligned}
$$

\section{A Preservation of the ordering of suprema}

In the proof of Theorem 2 we had to invoke Postulate 4, which says that the ordering of history-relative suprema is preserved across histories. Here we will show that Postulate 4 is not a consequence of the other Postulates of branching space-times. In the following section we will then show that Postulate 4 is a consequence of branching space-times theory augmented by a notion of "same space-time point". ${ }^{13}$

We will construct a model with two histories, $h_{1}$ and $h_{2}$. Let $\left\langle\mathbb{R}^{2}, \leq_{M}\right\rangle$ be the two-dimensional Minkowskian plane, i.e., the set of points in the two-dimensional real plane with the Minkowskian ordering,

$$
(x, y) \leq_{M}\left(x^{\prime}, y^{\prime}\right) \quad \text { iff } \quad\left(x-x^{\prime}\right)^{2} \leq\left(y-y^{\prime}\right)^{2} \text { and } y \leq y^{\prime} .
$$

This will be our first history, $h_{1}$. In order to have branching and thus, a second history, we add the set

$$
F=\{(*, y) \mid y>0\},
$$

\footnotetext{
${ }^{13}$ The formulation of Postulate 4 is due originally to Weiner (1997). He also gave a rather simple model showing that the Postulate is not a consequence of branching space-times alone (Weiner, personal communication). The model presented here was derived independently. It emphasizes the fact that histories in a branching space-times model may be radically non-isomorphic.
} 


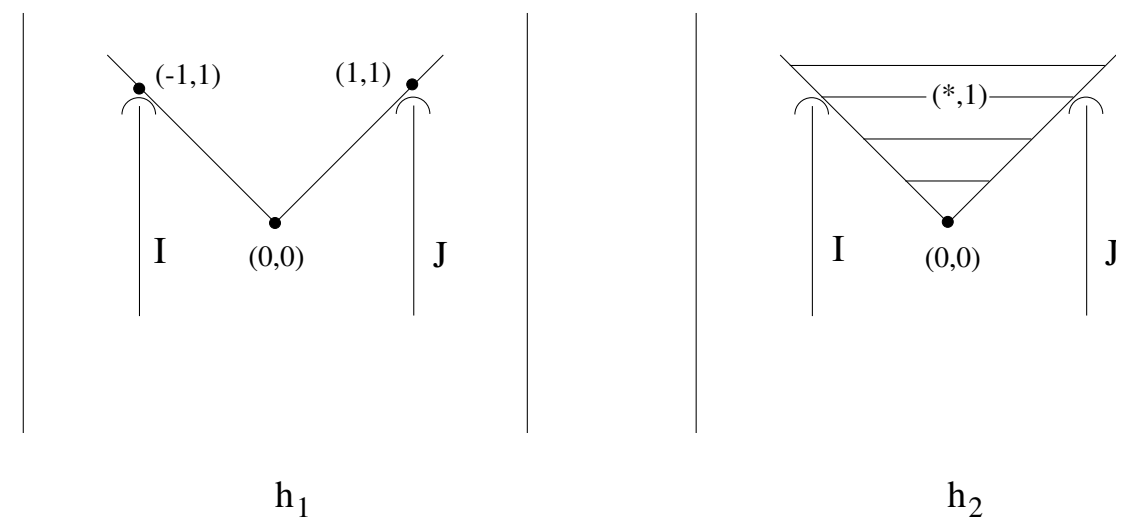

Figure 3: A branching space-times model violating Postulate 4. See text for details.

which is isomorphic to the positive reals. We now define the ordering $\leq$ on $W=$ $\mathbb{R}^{2} \cup F$ to be

$$
\begin{array}{lll}
(x, y) \leq\left(x^{\prime}, y^{\prime}\right) & \text { iff } & (x, y) \leq_{M}\left(x^{\prime}, y^{\prime}\right) \\
(*, y) \leq\left(*, y^{\prime}\right) & \text { iff } & y \leq y^{\prime} \\
(x, y)<\left(*, y^{\prime}\right) & \text { iff } & (x, y)<_{M}\left(-y^{\prime}, y^{\prime}\right) \text { or }(x, y)<_{M}\left(y^{\prime}, y^{\prime}\right) .
\end{array}
$$

Thus, for no $(x, y)$ and $\left(*, y^{\prime}\right)$ do we have $\left(*, y^{\prime}\right) \leq(x, y)$. In this way, $F$ is pasted into the future light cone above $(0,0)$. Through the ordering, $(*, y)$ is in some sense identified with the two points $(-y, y)$ and $(y, y)$ that are on the border of the future light cone above $(0,0)$. The resulting model is $\langle W, \leq\rangle$, pictured in Figure 3 . We need to establish that this is a model of branching space-times. Clearly, $W \neq \emptyset$.

Fact: The ordering is antisymmetric.

Proof: Since this is clear for the ordering on $\mathbb{R}^{2}$ and on $F$, we only need to look at the "mixed" case. But if $(x, y)<\left(*, y^{\prime}\right)$, it cannot be that $\left(*, y^{\prime}\right)<(x, y)$, by the definition of the ordering.

Fact: The ordering is transitive.

Proof: Select $a, b, c \in W$ such that $a<b, b<c$. If all the three points are in $\mathbb{R}^{2}$ or all in $F$, then $a<c$ follows immediately from transitivity of the respective orderings. So we only need to check two cases: (i) $a=(x, y), b=\left(x^{\prime}, y^{\prime}\right), c=$ $\left(*, y^{\prime \prime}\right)$. Assume that $b<c$ is due to the fact that $\left(x^{\prime}, y^{\prime}\right)<_{M}\left(y^{\prime \prime}, y^{\prime \prime}\right)$ (for $-y^{\prime \prime}$, argue analogously). Then $a<c$ follows from the transitivity of the Minkowskian ordering. (ii) $a=(x, y), b=\left(*, y^{\prime}\right), c=\left(*, y^{\prime \prime}\right)$. Assume that $(x, y)<\left(y^{\prime}, y^{\prime}\right)$ holds. Then since $y^{\prime}<y^{\prime \prime}$, in the Minkowskian ordering, $\left(y^{\prime}, y^{\prime}\right)<_{M}\left(y^{\prime \prime}, y^{\prime \prime}\right)$, and $a<c$ again follows from the transitivity of the Minkowskian ordering. 
Fact: Let $a=(x, y)>_{M}(0,0), b=\left(*, y^{\prime}\right)$. Then $a$ and $b$ are incomparable.

Proof: By the definition of the ordering.

Fact: In $\langle W, \leq\rangle$ there are exactly two histories.

Proof: $h_{1}=\mathbb{R}^{2}$ is a history, since it is upward directed, and by the previous fact, it cannot be extended by elements of $F$. The set

$$
h_{2}=\left(h_{1}-\left\{(x, y) \mid(0,0)<_{M}(x, y)\right\}\right) \cup F
$$

is also upward directed, and cannot be extended by elements of $\left\{(x, y) \mid(0,0)<_{M}\right.$ $(x, y)\}$ either. Again by the previous fact, "mixed" cases are excluded.

Fact: The ordering is dense and has infima and history-relative suprema.

Proof: From the properties of the orderings on $\mathbb{R}^{2}$ and on $F$.

Fact: The prior choice principle holds. There is exactly one splitting point, $(0,0)$. Proof: Let $c_{1}$ be a chain in $h_{1}-h_{2}$, i.e., in the future light cone of $(0,0)$. Then $(0,0)<c_{1}$, and $(0,0)$ is maximal in $h_{1} \cap h_{2}$. Alternatively, let $c_{2}$ be a chain in $h_{2}-h_{1}$. Again $(0,0)<c_{2}$, since $(0,0)<(*, y)$ for all $y>0$.

We have thus established that $\langle W, \leq\rangle$ is a model of branching space-times, respecting the Postulates (cf. Belnap 2002). We now prove that $\langle W, \leq\rangle$ violates Postulate 4.

Fact: In $W$, there are two initial chains $I, J$ that have different suprema in $h_{1}$, but the same supremum in $h_{2}$.

Proof: Let $I=\{(1,1-1 / n) \mid n \in N\}, J=\{(-1,1-1 / n) \mid n \in N\}$, as shown in Figure 3. These chains are in $h_{1} \cap h_{2}$, and they are bounded - e.g., by $(1,1)$ and $(-1,1)$, resp., which are their suprema in $h_{1}$. In $h_{2}$, however, $\sup _{h_{2}}(I)=(*, 1)=$ $\sup _{h_{2}}(J)$. - Along the same lines, one can construct suprema that are space-like related in $h_{1}$, but comparable in $h_{2}$ (e.g., set $I^{\prime}=\{(2,2-1 / n) \mid n \in N\}$ ).

\section{B Branching space-times with space-time points}

If we have available a notion of "the same spacetime point" in a model of branching space-times, then Postulate 4 holds. We define the notion of "same space-time point" as follows:

Definition 21 (Branching space-times with spacetime points) A triple $\langle W, \leq, \mathrm{S}\rangle$ is a model of branching space-times with spacetime points (BST+S) iff $\langle W, \leq\rangle$ is a model of branching space-times and $\mathrm{S}$ is an equivalence relation on $W$ such that

1. For each history $h$ in $W$ and for each equivalence class $[s], s \in W$, the intersection $h \cap[s]$ contains exactly one element. 
2. S respects the ordering, i.e., for $[s],\left[s^{\prime}\right]$ equivalence classes and $h_{1}, h_{2}$ histories, $[s] \cap h_{1}=\left[s^{\prime}\right] \cap h_{1}$ iff $[s] \cap h_{2}=\left[s^{\prime}\right] \cap h_{2}$, and the same for " $<$ " and for " $>$ ".

In $\mathrm{BST}+\mathrm{S}$ we can prove that history-relative suprema of initial chains, guaranteed to exist by BST, are at the same location.

Lemma 9 Let $\langle W, \leq, \mathrm{S}\rangle$ be a model of $B S T+S$, let I be an upper bounded chain in $W$, and let $h_{1}, h_{2}$ be two histories with $I \subseteq h_{1} \cap h_{2}$. Then $\mathrm{S}\left(\sup _{h_{1}}(I), \sup _{h_{2}}(I)\right)$.

Proof: Let $s_{1}=\sup _{h_{1}}(I)$, and let $s_{2}=\left[s_{1}\right] \cap h_{2}$ be the spacetime point in $h_{2}$ that is at the same position as $s_{1}$ according to $\mathrm{S}$. We need to prove that $s_{2}=\sup _{h_{2}}(I)$. First, $s_{2}$ is an upper bound for $I$ : Take $e \in I$. Since $e \leq s_{1}$ and $\mathrm{S}$ preserves order and $e \in h_{1} \cap h_{2}$, we have $e<s_{2}$. It remains to prove that $s_{2}$ is the smallest upper bound for $I$ in $h_{2}$. Assume otherwise. Then there has to be $s_{2}^{\prime} \in h_{2}, s_{2}^{\prime}<s_{2}$, and $s_{2}^{\prime}$ an upper bound for $I$. By the argument just given, $s_{1}^{\prime}=\left[s_{2}^{\prime}\right] \cap h_{1}$ is an upper bound for $I$ in $h_{1}$, and from order preservation, $s_{1}^{\prime}<s_{1}$. This contradicts the assumption that $s_{1}$ is the supremum (least upper bound) of $I$ in $h_{1}$. Thus, $s_{2}=\sup _{h_{2}}(I)$ : the supremum of $I$ is at the same spacetime point across histories.

As a corollary, we get that a model of BST $+\mathrm{S}$ satisfies Postulate 4.

\section{Corollary 1 In a model of $B S T+S$, Postulate 4 is satisfied}

Proof: Let $I, J$ be two initial chains, and let $h_{1}, h_{2}$ be two histories two which both $I$ and $J$ belong. Then by Lemma 9 , the history-relative suprema will be at the same spacetime points, and the claim follows by order preservation of $\mathrm{S}$.

With the model of Appendix A we have established the following:

Fact: Not every model of BST can be extended to a model of BST+S.

Proof: By the model from Appendix A and by corollary 1, using contraposition.

\section{References}

Aspect, A., Dalibard, J., and Roger, G. (1982). Experimental test of Bell's inequalities using time-varying analyzers. Physical Review Letters, 49:1804-1807.

Bell, J. (1987). Speakable and Unspeakable in Quantum Mechanics: Collected Papers on Quantum Philosophy. Cambridge: Cambridge University Press.

Belnap, N. (1992). Branching space-time. Synthese, 92:385-434. 
Belnap, N. (2002). EPR-like "funny business" in the theory of branching spacetimes. In Placek, T. and Butterfield, J., eds., Non-locality and Modality, p. 293315. Dordrecht: Kluwer.

Belnap, N. (2003a). No-common-cause EPR-like funny business in branching space-times. Philosophical Studies, 114:199-221.

Belnap, N. (2003b). A theory of causation: Causae causantes (originating causes) as inus conditions in branching space-times. British Journal for the Philosophy of Science, forthcoming.

Jensen, F. V. (2001). Bayesian Networks and Decision Graphs. Berlin: Springer.

Pearl, J. (2000). Causality. Cambridge: Cambridge University Press.

Popper, K. (1982). Quantum Theory and the Schism of Physics. London: Hutchinson.

Reichenbach, H. (1956). The Direction of Time. Berkeley, CA: University of California Press.

van Fraassen, B. (1980). The Scientific Image. Oxford: Oxford University Press.

von Wright, G. H. (1963). Norm and Action. A Logical Inquiry. London: Routledge.

Weiner, M. (1997). Probability in branching space-time. Unpublished. Department of Philosophy, University of Pittsburgh.

Weiner, M. and Belnap, N. (2004). How causal probabilities might fit into our objectively indeterministic world. Synthese, forthcoming. 